hep-th/0308037

PUPT-2093

\title{
Chiral Rings, Vacua and Gaugino Condensation of Supersymmetric Gauge Theories
}

\author{
Peter Svrcek \\ Joseph Henry Laboratories, Princeton University \\ Princeton, New Jersey 08544, USA
}

We find the complete chiral ring relations of the supersymmetric $U(N)$ gauge theories with matter in adjoint representation. We demonstrate exact correspondence between the solutions of the chiral ring and the supersymmetric vacua of the gauge theory. The chiral ring determines the expectation values of chiral operators and the low energy gauge group. All the vacua have nonzero gaugino condensation. We study the chiral ring relations obeyed by the gaugino condensate. These relations are generalizations of the formula $S^{N}=\Lambda^{3 N}$ of the pure $\mathcal{N}=1$ gauge theory.

August 2003 


\section{Introduction}

Recently there has been a progress in understanding the dynamics a wide class of supersymmetric field theories. Embedding of the gauge theories in string theory as low energy effective field theories of D branes wrapped on cycles in Calaby-Yau threefolds led to the conjecture of Dijkgraaf and Vafa that holomorphic data of the field theories can be calculated from an auxiliary matrix model. The bosonic potential of the matrix model is the superpotential of the gauge theory. Cachazo, Douglas, Seiberg and Witten gave a field theory derivation the results that rests on the analysis of the anomalies and of the ring of chiral operators of the field theory.

It has been known for over a decade that the chiral ring of two dimensional field theories determines the structure of its supersymmetric vacua. The chiral operators obey relations that hold in every supersymmetric vacuum of the theory. It has been shown in 11] for the $\mathcal{N}=2$ superconformal field theories and in [2] for the $C P^{N-1}$ supersymmetric sigma model that there is an exact correspondence between the solutions to the chiral ring relations and the supersymmetric vacua of the theory.

The authors of [3] showed that this continues to hold in four dimensions for the $\mathcal{N}=1$ pure $U(N)$ gauge theory. In this article we will extend this correspondence for $\mathcal{N}=1 U(N)$ gauge theories with matter field $\Phi$ in the adjoint representation. The adjoint field has superpotential superpotential

$$
W(\Phi)=\sum_{k=0}^{n} \frac{g_{k}}{k+1} \operatorname{Tr} \Phi^{k+1} .
$$

We can view this theory as a deformation of the $\mathcal{N}=2$ gauge theory by the superpotential (1.1) for the adjoint scalar $\Phi$ of the $\mathcal{N}=2$ vector superfield.

We will show that solving the chiral ring equations is equivalent to factorization of the $\mathcal{N}=2$ curve. The factorization was originally derived by a strong coupling analysis of the gauge theory [4] based on monopole condensation.

\section{Summary of Results}

In section 2, we review the general properties of chiral rings, their relation to supersymmetric vacua and discuss the chiral ring relations both on the classical and quantum level. In section 3, we solve the chiral ring relations and demonstrate exact correspondence between the supersymmetric vacua and the roots of the chiral ring relations. In section 4 , we use the chiral ring relations to give a brief discussion of the intersection of the vacua. In section 5, we study the chiral ring relations obeyed by the gaugino condensate and in section 6 we treat examples that illustrate the results from previous sections. 


\section{The Chiral Ring}

Chiral operators are the operators that are annihilated by the antichiral supersymmetry generators $\bar{Q}_{\dot{\alpha}}$. Instead of chiral operators we will consider the set equivalence classes of chiral operators where two operators are in the same equivalence class if they differ by a term of the form $\left\{\bar{Q}_{\dot{\alpha}}, \ldots\right\}$. This set is a ring because the product of two equivalence classes of chiral operators is another equivalence class. The expectation value of a chiral operator in a supersymmetric vacuum depends only on its equivalence class because the vacuum is annihilated by the supersymmetry generator $\bar{Q}_{\dot{\alpha}}$. It follows from $\left\{Q_{\alpha}, \bar{Q}_{\dot{\alpha}}\right\}=2 \sigma_{\alpha \dot{\alpha}}^{\mu} P_{\mu}$ that momentum, which is the generator of translations, annihilates chiral operators. Hence, chiral operators are independent of position. The chiral ring keeps only the information about the zero modes. In a product of chiral operators, we can put the operators far apart without changing the value of the product. Then the product factorizes into individual operators by cluster decomposition principle. Hence, we need to consider only the single trace operators. To classify the single trace operators we notice the identity [3]

$$
\left[\bar{Q}^{\dot{\alpha}}, D_{\alpha \dot{\alpha}} \mathcal{O}\right\}=\left[W_{\alpha}, \mathcal{O}\right\}
$$

which holds for any adjoint valued chiral superfield. Substituting $\Phi$ for $\mathcal{O}$, we see that $\Phi$ commutes with $W_{\alpha}$

$$
\left[\Phi, W_{\alpha}\right]=0
$$

so it suffices to consider only operators where all $\Phi$ 's are grouped together. Taking $\mathcal{O}=W_{\alpha}$ in (2.1) we learn that $W_{\alpha}$ 's anticommute

$$
\left\{W_{\alpha}, W_{\beta}\right\}=0
$$

It follows that the single trace operators with three or more gaugino operators are descendants because the fermionic index $\alpha$ takes two values. The single trace chiral operators are

$$
\begin{aligned}
u_{k} & =\operatorname{Tr} \Phi^{k}, \\
w_{\alpha, k} & =\frac{1}{4 \pi} \operatorname{Tr} \Phi^{k} W_{\alpha}, \\
r_{k} & =\frac{-1}{32 \pi^{2}} \operatorname{Tr} \Phi^{k} W_{\alpha} W^{\alpha} .
\end{aligned}
$$


We assemble these operators into the resolvents

$$
\begin{aligned}
T(z) & =\operatorname{Tr} \frac{1}{z-\Phi}=\sum_{k \geq 0} u_{k} z^{-1-k} \\
w_{\alpha}(z) & =\frac{1}{4 \pi} \operatorname{Tr} W_{\alpha} \frac{1}{z-\Phi}=\sum_{k \geq 0} w_{\alpha, k} z^{-1-k} \\
R(z) & =-\frac{1}{32 \pi^{2}} \operatorname{Tr} W_{\alpha} W^{\alpha} \frac{1}{z-\Phi}=\sum_{k \geq 0} r_{k} z^{-1-k} .
\end{aligned}
$$

The single trace operators $u_{k}, w_{\alpha, k}$ and $r_{k}$ generate the chiral ring. Formally, the chiral ring is a polynomial ring over the field of complex number with the single trace operators as indeterminates

$$
\mathcal{C}=C\left[u_{k}, w_{\alpha, k}, r_{k}\right]
$$

Our interest is in the relations that the chiral operators satisfy. These relations are operator statements that hold in any supersymmetric vacuum. By taking an expectation value of a chiral ring relation in a given vacuum and using the fact that the expectation value of a product of chiral operators factorizes we get a relation for the expectation values of the chiral operators in that particular vacuum. By solving the chiral ring we mean finding the solutions to these chiral ring equations. The vacuum expectation values of $u_{k}, w_{\alpha, k}, r_{k}$ in a supersymmetric vacuum solve the chiral ring relations by definition. In principle, the chiral ring relations could have additional "unphysical" solutions for $u_{k}, w_{\alpha, k}, r_{k}$ which do not correspond to any supersymmetric vacuum. We will show that this is not the case. The roots of the chiral ring relations are in exact correspondence with the supersymmetric vacua of the gauge theory.

We can make the correspondence more precise. We introduce further algebraic construct, the coordinate chiral ring, which is the quotient of the chiral ring by the ideal of the chiral ring relations. Two chiral operators are considered to be the same elements of the coordinate chiral ring if their difference is a chiral ring relation. Hence, the coordinate chiral ring encodes the information about chiral operators that is invariant under addition of chiral ring relations. There is a natural correspondence between the roots of the chiral ring relations and the elements of the coordinate chiral ring. For semisimple coordinate chiral ring, all the roots are single and isolated, the only information that the coordinate ring encodes is the value of the operators at the solutions of the chiral ring relations. The solutions correspond to idempotent elements of the coordinate chiral ring. An idempotent is an operator that squares to itself. The idempotent associated to a particular vacuum 
takes expectation value one in that vacuum and vanishes in other vacua. In the general case, the roots can be multiple or have massless fermionic directions for the $U(1)$ photinos. Then a root corresponds to an ideal, called local ring, of the coordinate ring generated by the idempotent element above. The local ring is the set of elements obtained by multiplying the idempotent by all chiral operators. The dimension of the local ring equals the multiplicity of the corresponding vacuum. The basis of the local ring consists of the idempotent together with nilpotent elements. The coordinate chiral ring is a direct sum of the local rings. Any operator can be expanded as

$$
\mathcal{O}=\sum_{i} o_{i} \Pi_{i}+n_{i}
$$

where $\Pi_{i}$ are the idempotents corresponding to $i$ 'th vacuum and $n_{i}$ is the nilpotent part of $\mathcal{O}$ in the $i$ 'th local ring. The nilpotent elements correspond to different intersecting vacua or to vacuum with different value of the nilpotent $U(1)$ photinos $\operatorname{Tr} \Phi^{k}$. The expectation value of an operator does not depend on these parameters hence it does not depend on the nilpotent part $n_{i}$. The expectation value of $\mathcal{O}$ in the $i$ 'th group of vacua is $o_{i}$.

Hence, each supersymmetric vacuum corresponds to a solution of the chiral ring relations which naturally corresponds to local ring which is generated by an idempotent element together with nilpotents. This allows us to calculate the expectation values of the chiral operators from the knowledge of the idempotents.

A simple example that illustrates the above discussion in the case of isolated vacua is the polynomial ring in one indeterminate $C[x]$. This is the case of $U(1)$ gauge theory, $x$ is the $1 \times 1$ matrix $\Phi$ in the adjoint representation of $U(1)$ which is trivial. The $n$ vacua of the theory are at the critical points of the superpotential $W^{\prime}(\Phi)=\prod_{i=1}^{n}\left(\Phi-\lambda_{i}\right)$. Hence, the indeterminate $x$ satisfies the polynomial relation of $n^{\prime}$ th degree $W^{\prime}(x)=0$. The coordinate chiral ring

$$
C[x] /\left(W^{\prime}(x)=0\right)
$$

has dimension $n$. The $n$ distinguished idempotents are

$$
\Pi_{i}(x)=\prod_{j \neq i}\left(x-\lambda_{j}\right) / \prod_{j \neq i}\left(\lambda_{i}-\lambda_{j}\right) .
$$

Clearly $\Pi_{i}$ takes value one at $\lambda_{i}$ and vanishes at $\lambda_{j}$ for $j \neq i$. Any polynomial of degree less than $n$ can be expressed as a linear combination of $\Pi(x)$. For polynomials of a higher degree, we reduce the degree below $n$ using the relation $W^{\prime}(x)=0$. This completes the 
proof that the idempotents $\Pi_{i}(x)$ form an $n$ dimensional basis of the coordinate chiral ring. The expansion coefficients of a polynomial

$$
S(x)=\sum s_{i} \Pi_{i}(x)
$$

in the idempotents are the values that the polynomial takes at the $n$ roots of $W^{\prime}(x)$

$$
S\left(\lambda_{i}\right)=\sum_{k} s_{k} \Pi_{k}\left(\lambda_{i}\right)=s_{i}
$$

in agreement with our general discussion.

To illustrate the correspondence when the coordinate ring has nilpotent elements, we consider the polynomial ring in one indeterminate $x$ which satisfies $x^{n}=0$. This is the case of $n$ intersecting vacua of the field $\Phi$. The coordinate chiral ring

$$
C[x] /\left(x^{n}=0\right)
$$

is an $n$ dimensional complex vector space. The basis consists of the idempotent 1 and of the nilpotents $x, x^{2}, \ldots, x^{n-1}$. Any polynomial can be expanded in this basis modulo the relation $x^{n}=0$ which eliminates the powers of $x^{k}$ for $k \geq n$. The value of the polynomial at the root $x=0$ equals the $x^{0}$ order coefficient which is coefficient the idempotent 1 in the expansion of the polynomial in terms of the above basis. Hence, to find the expectation value of a chiral operator we expand it in the basis of the coordinate chiral ring and read off the coefficient at the idempotent element. Chiral operators have the same expectation value in each of the intersecting vacua. The $n$ intersecting vacua correspond to the multiple root which in turn corresponds to the $n$ dimensional coordinate chiral ring that is spanned by the idempotent and nilpotent elements.

We can view the quantum relations as deformations of the classical relations. The classical relations can receive both perturbative and nonperturbative corrections. Quantum generalization of the classical equations of motion are the perturbative Ward identities coming from one-loop Konishi anomaly. The $\operatorname{Tr} \Phi^{k}$ with $k>N$ can be expressed as a polynomial in $u_{1}, \ldots, u_{N}$ because an $N \times N$ matrix is specified by the $N$ independent gauge invariant operators $u_{1}, \ldots, u_{N}$. The classical relations for $\operatorname{Tr} \Phi^{k}$ are deformed nonperturbatively by instanton corrections. 


\subsection{Perturbative Corrections}

In this subsection we will find the classical chiral ring relations that follow from equations of motion and review the anomaly that corrects these relations. We start by multiplying the classical equation of motion for $\Phi$,

$$
\partial_{\Phi} W(\Phi)=\bar{D}_{\dot{\alpha}} \bar{D}^{\dot{\alpha}} \bar{\Phi}
$$

with $A /(z-\Phi)$ where $A=1, \frac{1}{4 \pi} W_{\alpha}$ or $-\frac{1}{32 \pi^{2}} W_{\alpha} W^{\alpha}$ and take the trace

$$
\operatorname{Tr} A \frac{W^{\prime}(\Phi)}{z-\Phi}=0
$$

We used the fact that $\bar{D}_{\dot{\alpha}}$ is conjugate to $\bar{Q}_{\dot{\alpha}}$ hence the right hand side of (2.14) can be written as $\left\{\bar{Q}_{\alpha}, \ldots\right\}$ and is a chiral ring descendant. To express these equations in terms of the resolvents (2.5), we notice the following identity

$$
\begin{aligned}
\operatorname{Tr} A \frac{W^{\prime}(\Phi)}{z-\Phi} & =W^{\prime}(z) \operatorname{Tr} \frac{A}{z-\Phi}-\operatorname{Tr} A \frac{\left(W^{\prime}(z)-W^{\prime}(\Phi)\right)}{z-\Phi} \\
& =W^{\prime}(z) \operatorname{Tr} \frac{A}{z-\Phi}-a(z) .
\end{aligned}
$$

The function $a(z)$ is a polynomial in $z$ of degree $n-1$ because $W^{\prime}(z)-W^{\prime}(\Phi)$ is a polynomial in $z$ of degree $n$ that vanishes when $z$ equals to one of the eigenvalues of $\Phi$. We define the polynomials

$$
\begin{aligned}
f(z) & =\frac{4}{32 \pi^{2}} \operatorname{Tr} W_{\alpha} W^{\alpha} \frac{W^{\prime}(z)-W^{\prime}(\Phi)}{z-\Phi}, \\
\rho_{\alpha}(z) & =\frac{1}{4 \pi} \operatorname{Tr} W_{\alpha} \frac{W^{\prime}(z)-W^{\prime}(\Phi)}{z-\Phi}, \\
c(z) & =\operatorname{Tr} \frac{W^{\prime}(z)-W^{\prime}(\Phi)}{z-\Phi}
\end{aligned}
$$

and rewrite (2.14) with the help of (2.15) in the form

$$
\begin{aligned}
& 0=W^{\prime}(z) R(z)+\frac{1}{4} f(z), \\
& 0=W^{\prime}(z) w_{\alpha}(z)-\rho_{\alpha}(z), \\
& 0=W^{\prime}(z) T(z)-c(z) .
\end{aligned}
$$

To find the quantum corrections to (2.17) we recall that the classical equations of motions are derived by varying $\Phi$ in the action. We will now review the anomaly in the 
variation which corrects the above relations quantum mechanically. Varying $\Phi$ by a general holomorphic function $\delta \Phi=f\left(\Phi, W_{\alpha}\right)$ gives anomaly of the current

$$
J_{f}=\operatorname{Tr} \bar{\Phi} e^{\operatorname{ad} V} f\left(\Phi, W_{\alpha}\right)
$$

which generates the variation of $\Phi$. We find

$$
\bar{D}_{\alpha} \bar{D}^{\alpha} J_{f}=\operatorname{Tr} f\left(\Phi, W_{\alpha}\right) \frac{\partial W(\Phi)}{\partial \Phi}+\text { anomaly }+\bar{D}(\ldots)
$$

The first term on right hand side is the classical variation. The anomaly comes from oneloop diagrams involving $\bar{\Phi}$ and a single $\Phi$ from $f\left(\Phi, W_{\alpha}\right)$. To find the generalized Konishi equations expressed in terms of the resolvents (2.5) we make the variation

$$
\delta \Phi_{i j}=f\left(\Phi, W_{\alpha}\right)_{i j}=\left(\frac{A}{z-\Phi}\right)_{i j}
$$

Computing the anomaly and setting $\left\{\bar{D}_{\dot{\alpha}}, \ldots\right\}$ terms to zero gives [3]

$$
\begin{aligned}
R^{2}(z) & =W^{\prime}(z) R(z)+\frac{1}{4} f(z), \\
2 R(z) w_{\alpha}(z) & =W^{\prime}(z) w_{\alpha}(z)-\rho_{\alpha}(z), \\
2 R(z) T(z)+w_{\alpha}(z) w^{\alpha}(z) & =W^{\prime}(z) T(z)-c(z) .
\end{aligned}
$$

On the left hand side of the anomaly equations are the classical equations of motion (2.17) and on the right hand side are the perturbative corrections coming from the one-loop Konishi anomaly. We can solve the anomaly equations (2.21) for the resolvents $R(z), w_{\alpha}(z)$ and $T(z)$ in terms of the superpotential and the auxiliary polynomials

$$
\begin{aligned}
R(z) & =\frac{1}{2}\left(W^{\prime}(z)-\sqrt{W^{\prime 2}(z)+f(z)}\right) \\
w_{\alpha}(z) & =\frac{\rho_{\alpha}(z)}{\sqrt{W^{\prime 2}(z)+f(z)}} \\
T(z) & =\frac{c(z)+w_{\alpha}(z) w^{\alpha}(z)}{\sqrt{W^{\prime 2}(z)+f(z)}} .
\end{aligned}
$$

Throughout most of the article we will neglect the quadratic term $w_{\alpha}(z) w^{\alpha}(z)$ in the relation for $T(z)$. 


\subsection{Nonperturbative Corrections}

The gauge invariant operators $u_{k}=\operatorname{Tr} \Phi^{k}$ obey relations coming from the fact that $\Phi$ is an $N \times N$ matrix. $\Phi$ is determined up to a gauge transformation by the independent gauge invariant operators $\operatorname{Tr} \Phi^{l}$ with $l=1, \ldots, N$. The operators $\operatorname{Tr} \Phi^{k}$ with $k>N$ can be expressed as polynomials in the first $N$ traces. We will find the classical formulas for $\operatorname{Tr} \Phi^{k}$ and then we will show how they get modified by nonperturbative instanton corrections.

$\Phi$ can be specified by the characteristic polynomial of $N^{\prime}$ th degree

$$
P(z)=\operatorname{det}(z-\Phi)=\prod_{i=1}^{N}\left(z-\lambda_{i}\right)
$$

The roots of $P(z)$ are the classical eigenvalues $\lambda_{i}$ of $\Phi$. We refer the reader to Appendix A. for more details on $P(z)$. To derive the relations for $u_{k}$ we write the generating function $T(z)$ in terms of eigenvalues of $\Phi$

$$
T(z)=\operatorname{Tr} \frac{1}{z-\Phi}=\sum_{i=1}^{N} \frac{1}{z-\lambda_{i}}
$$

and notice that this is the same as $P^{\prime}(z) / P(z)$. Hence we have

$$
T(z)=\frac{P^{\prime}(z)}{P(z)} \text {. }
$$

Notice that the left hand side depends on all traces $\operatorname{Tr} \Phi^{k}=u_{k}$ while the right hand side depends only on $u_{1}, \ldots, u_{N}$. Expanding (2.25) in powers of $1 / z$ and comparing the coefficients of $z^{-k-1}$ we get an expression for $u_{k}$ from left hand side as a polynomial in $u_{1}, \ldots, u_{N}$ from the right hand side. We give a few examples of the resulting formulas in the Appendix A.

The operators

$$
m_{k}=\operatorname{Tr} M \Phi^{k}=\sum_{i=1}^{N} M_{i i} \lambda_{i}^{k}
$$

where $\mathrm{M}$ is an arbitrary $N \times N$ matrix depend on $2 N$ parameters, the $N$ eigenvalues of $\Phi$ and the $N$ diagonal elements of $M$. Hence, $m_{0}, m_{1}, \ldots m_{N-1}$ together with $u_{1}, u_{2}, \ldots, u_{N}$ are independent variables that determine $m_{k}$ for $k \geq N$. To find the relations for $m_{k}$ we make first order variation of (2.25) as $\Phi^{\prime}=\Phi+\epsilon M$

$$
\operatorname{Tr} \frac{M}{(z-\Phi)^{2}}=-\frac{P_{m}^{\prime}(z)}{P(z)}+\frac{P_{m}(z) P^{\prime}(z)}{P^{2}(z)}=-\left(\frac{P_{m}(z)}{P(z)}\right)^{\prime} .
$$


The characteristic polynomial $P_{m}(z)$ of degree $N-1$ comes from the first variation of $P(z)$ in $M$

$$
P_{m}(z)=-\left.\partial_{\epsilon} \operatorname{det}(z-\Phi-\epsilon M)\right|_{\epsilon=0}=\sum_{i=1}^{N} M_{i i} \prod_{j \neq i}\left(z-\lambda_{j}\right) .
$$

We integrate (2.27) to get

$$
\operatorname{Tr} \frac{M}{z-\Phi}=\frac{P_{m}(z)}{P(z)} .
$$

We fixed the constant of integration by requiring both sides of the equation to fall off to zero for $z$ going to infinity. The left hand side of (2.29) depends on $m_{k}$ while the right hand side depends only on $m_{0}, m_{1}, \ldots, m_{N-1}$ and $u_{1}, u_{2}, \ldots, u_{N}$. Expanding (2.29) in $1 / z$ and comparing the coefficients of $z^{-k-1}$ term we find formula for $m_{k}$ as a polynomial in $m_{0}, m_{1}, \ldots, m_{N-1}$ and $u_{1}, u_{2}, \ldots, u_{N}$ from the right hand side.

We find the classical relations for $w_{\alpha, k}$ and $r_{k}$ by substituting $\frac{1}{4 \pi} W_{\alpha}$ and $-\frac{1}{32 \pi^{2}} W_{\alpha} W^{\alpha}$ for $M$ in $(2.29)$

$$
\begin{aligned}
& w_{\alpha}(z)=\frac{P_{\alpha}(z)}{P(z)} \\
& R(z)=\frac{P_{R}(z)}{P(z)} .
\end{aligned}
$$

We have mentioned that the classical relations for $\operatorname{Tr} \Phi^{k}$ with $k>N$ receive instanton corrections. We will determine the quantum modified relations by strong coupling analysis by considering the $\mathcal{N}=1$ theory as a deformation of the $\mathcal{N}=2$ theory by a superpotential for the adjoint scalar field [3,4]. We will closely follow [3] but we will consider a vacuum with nonzero expectation value of the $U(1)$ photinos $\operatorname{Tr} W_{\alpha} \Phi^{k}$ which break the $\mathcal{N}=2$ supersymmetry to $\mathcal{N}=1$ even before we turn on the superpotential. The superpotential takes the form

$$
W^{\prime}(z)=g P(z)=g \prod_{i=1}^{N}\left(z-\lambda_{i}\right)
$$

with all $\lambda_{i}$ different. We the maximally Higgsed vacuum in which the eigenvalues of $\Phi$ to occupy the $N$ different critical points of the superpotential. $\Phi$ breaks the $U(N)$ gauge group down to $U(1)^{N}$. To find the resolvents (2.22)

$$
\begin{aligned}
R(z) & =\frac{1}{2}\left(g P(z)-\sqrt{g^{2} P^{2}(z)+f(z)}\right) \\
w_{\alpha}(z) & =\frac{\rho_{\alpha}(z)}{\sqrt{g^{2} P^{2}(z)+f(z)}} \\
T(z) & =\frac{c(z)}{\sqrt{g^{2} P^{2}(z)+f(z)}}
\end{aligned}
$$


we need to determine the auxiliary polynomials $f(z), c(z), \rho_{\alpha}(z)$. The polynomial $c(z)$ depends on the operators $u_{k}$ for $k=1, \ldots, N-1$

$$
c(z)=\operatorname{Tr} \frac{W^{\prime}(z)-W^{\prime}(\Phi)}{z-\Phi}=g \sum_{i=1}^{N} \frac{P(z)-P\left(\lambda_{i}\right)}{z-\lambda_{i}}=g P^{\prime}(z) .
$$

The third equality follows from the special form of the superpotential (2.32). We find $f(z)$ by comparing the $\mathcal{N}=2$ curve [3]

$$
y^{2}=P^{2}(z)-4 \Lambda^{2 N}
$$

with the matrix model curve

$$
y_{m}^{2}=W^{\prime 2}(z)+f(z) .
$$

Each of the occupied critical points of the superpotential gets smeared into a cut. Hence, the matrix model curve has single roots only. We find it from the $\mathcal{N}=2$ curve by factoring out the double roots

$$
P^{2}(z)-4 \Lambda^{2 N}=Q^{2}(z)\left(g^{2} P^{2}(z)+f(z)\right) .
$$

In the present case, there are no double roots so $Q(z)=1 / g, f(z)=-4 g^{2} \Lambda^{2 N}$. Substituting $c(z)$ and $f(z)$ into (2.33) we get the quantum modified versions of the formula (2.25) for $T(z)$

$$
T(z)=\frac{P^{\prime}(z)}{\sqrt{P^{2}(z)-4 \Lambda^{2 N}}} .
$$

This relation is valid for the $\mathcal{N}=2$ gauge theory because it does not depend on $g$ so we might as well set $g$ to zero restoring $\mathcal{N}=2$ supersymmetry.

For general superpotential, we argue that (2.38) continues to hold. We again use the factorization of the $\mathcal{N}=2$ curve. In general, some number, say $h$ of the critical points of the superpotential are unoccupied. The corresponding roots of the curve $y^{2}=W^{\prime 2}(z)+f(z)$ do not get smeared into cuts, they remain double roots. The matrix model curve sees only the $N-h$ occupied critical points, hence we factor out the double roots

$$
H^{2}(z) y_{m}^{2}=W^{\prime 2}(z)+f(z)
$$

The roots of $H(z)$ are near the unoccupied critical points of the superpotential. They are moved from the classical critical points by gaugino condensation, which is encoded in the 
polynomial $f(z)$. Factoring out the double roots of the $\mathcal{N}=2$ curve we get the matrix model curve

$$
\left(P^{2}(z)-4 \Lambda^{2 N}\right)=Q^{2}(z) y_{m}^{2}(z)
$$

Taking first derivative of this equation we see that $P^{\prime}(z)$ is divisible by $Q(z)$. Hence, we write $P^{\prime}(z)=Q(z) \tilde{P}(z)$. Furthermore, $c(z)$ must be divisible by $H(z)$. Otherwise

$$
T(z)=\frac{c(z)}{H(z) y_{m}(z)}
$$

would have poles at the roots of $H(z)$ which is a contradiction. The number of eigenvalues of $\Phi$ at the $i$ 'th critical point is

$$
N_{i}=\frac{1}{2 \pi i} \oint_{C_{i}} d z T(z)=\frac{1}{2 \pi i} \oint_{C_{i}} d z \frac{c(z)}{H(z) y_{m}(z)}
$$

where $C_{i}$ is a curve going counterclockwise around the $i$ 'th critical point. The occupation number vanishes for the unoccupied critical points. Hence, $T(z)$ cannot have a pole at the unoccupied critical point because the contour integral would pick out the residue $T(z)$ and give a nonzero answer for $N_{i}$. So, $c(z)=H(z) \tilde{c}(z)$.

The $N_{i}$ 's can be also calculated as the logarithmic residues of $P(z)$. For small $\Lambda, N_{i}$ roots of $P(z) \sim \prod_{i=1}^{N}\left(z-\lambda_{i}\right)$ are near the classical critical points of the superpotential. For small $\Lambda$, the integral

$$
\oint d z \frac{P^{\prime}(z)}{P(z)}=\oint d z \ln ^{\prime}(P(z))
$$

counts the number of roots of $P(z)$ near the $i$ 'th classical critical point of the superpotential. This is the same as the number of eigenvalues of $\Phi$ at that critical point. When we turn on $\Lambda$, and deform the contour $C_{i}$ so that none of the roots of $P(z)$ cross it, (2.43) is still valid. We can deform this formula even more to

$$
N_{i}=\oint_{C_{i}} d z \frac{P^{\prime}(z)}{\sqrt{P^{2}(z)-\Lambda^{2 N}}}
$$

again making sure that the contour $C_{i}$ does not cross the cuts of the square root in the denominator. We get an integer answer which must be $N_{i}$ by continuity. Hence, we have two equivalent formulas for the occupation numbers

$$
N_{i}=\oint_{C_{i}} d z \frac{\tilde{c}(z)}{y_{m}(z)}=\oint_{C_{i}} d z \frac{\tilde{P}(z)}{y_{m}(z)}
$$


The polynomials $\tilde{P}(z)$ and $\tilde{c}(z)$ have the same degree $N-h-1$. The $N-h$ equations coming from (2.45) for the $N-h$ coefficients of $\tilde{c}(z)$ or $\tilde{P}(z)$ uniquely determine these two polynomials. Hence they are the same as polynomials in $z$ but at the same time they depend on the vacuum nontrivially through (2.45). Hence, (2.38) holds every vacuum of the gauge theory which means that it is a chiral ring relation.

To fix the formula for $w_{\alpha}(z)$ we still need to find the fermionic polynomial $\rho_{\alpha}(z)$. This goes through as in (2.34) thanks to the special form of the superpotential

$$
\rho_{\alpha}(z)=\operatorname{Tr} W_{\alpha} \frac{\left(W^{\prime}(z)-W^{\prime}(\Phi)\right)}{z-\Phi}=g \sum_{i=1}^{N} W_{i i} \frac{P(z)-P\left(\lambda_{i}\right)}{z-\lambda_{i}}=g P_{\alpha}(z) \text {. }
$$

$\rho_{\alpha}(z)$ has coefficients which are linear $w_{\alpha, k}$ and polynomials in $u_{k}$ with $k=1, \ldots, N-1$. For more details on $P_{\alpha}(z)$ we refer the reader to Appendix A. Substituting $\rho_{\alpha}(z)$ and $f(z)$ into (2.33) we get the quantum modified version of (2.30)

$$
w_{\alpha}(z)=\frac{P_{\alpha}(z)}{\sqrt{P^{2}(z)-4 \Lambda^{2 N}}} .
$$

The formula for $T(z)$ has been derived in [3,5] while the relation for $w_{\alpha}(z)$ is new. One can show that (2.47) holds for arbitrary superpotential similarly as we showed the validity of (2.38) in previous paragraph. By taking the superpotential to zero we learn that (2.47) is valid for the $\mathcal{N}=2$ gauge theory. We need to keep in mind that the chiral operators $\operatorname{Tr} W_{\alpha} \Phi^{k}$ are descendants of the $\mathcal{N}=2$ chiral ring [3] hence the formula for photinos makes sense only in the $\mathcal{N}=1$ chiral ring. A different reason for considering the formulas as an $\mathcal{N}=1$ chiral ring relation for the $\mathcal{N}=2$ gauge theory is that the VEV's of photinos break the $\mathcal{N}=2$ gauge symmetry down to $\mathcal{N}=1$. For the lack of a better name, we will call the relations coming from (2.47) and (2.38) the $\mathcal{N}=2$ relations. A suitable linear combination of the coeffients of $P_{\alpha}(z)$ are the $\mathcal{N}=2$ low energy photinos. Physically, the relations for gaugino operators describe the expectation value of the $w_{\alpha, i}$ 's when we turn on a coherent state of zero modes of the photinos.

It is easy to find now the formula for $R(z)$. We divide the third equation in (2.21) by $2 T(z)$ to get

$$
R(z)=-\frac{w_{\alpha}(z) w^{\alpha}(z)}{2 T(z)}+\frac{1}{2} W^{\prime}(z)-\frac{c(z)}{2 T(z)} .
$$

Substituting (2.38) and (2.47) into (2.48) we get

$$
R(z)=-\frac{P_{\alpha}(z) P^{\alpha}(z)}{2 P^{\prime}(z) \sqrt{P^{2}(z)-4 \Lambda^{2 N}}}+\frac{W^{\prime}(z)}{2}-c(z) \frac{\sqrt{P^{2}(z)-4 \Lambda^{2 N}}}{2 P^{\prime}(z)} .
$$


The first term on the right hand side represent the quadratic response of $R(z)$ to nonzero vacuum expectation value of the $\mathcal{N}=2$ photinos. The next terms are linear in the coefficients of the superpotential, as expected. They give the gaugino condensate of the $\mathcal{N}=1$ gauge theory with nonzero superpotential. Taking $\Lambda=0$ we get the classical relation

$$
R(z)=-\frac{P_{\alpha}(z) P^{\alpha}(z)}{2 P^{\prime}(z) P(z)}
$$

where we used that

$$
W^{\prime}(z) P^{\prime}(z)=c(z) P(z)
$$

holds in the classical chiral ring. This follows from combining the two relations (2.17) and (2.25) for $T(z)$. We have derived from classical considerations that $R(z)=P_{R}(z) / P(z)$. This agrees with (2.50) only if

$$
P_{R}(z) P^{\prime}(z)=-\frac{1}{2} P_{\alpha}(z) P^{\alpha}(z)
$$

We have not been able to verify this relation.

We recast the $\mathcal{N}=2$ relations into a different form that is more convenient for some applications. We integrate both sides of the equation (2.38) to get

$$
\int T(z)=\ln \frac{1}{2}\left(P(z)+\sqrt{P^{2}(z)-4 \Lambda^{2 N}}\right),
$$

where the integral means that we expand $T(z)$ in inverse powers of $z$ and then integrate the resulting series

$$
\int T(z)=\int d z \sum_{l=0}^{\infty} \frac{u_{l}}{l z^{l+1}}=N \ln (z)+\sum_{l=1}^{\infty} \frac{u_{l}}{z^{l}} .
$$

The constant of integration in 2.53) was determined by matching the $N \ln (z)$ terms on both sides of the equation. Finally, we can find $P(z)$ from (2.53),

$$
P(z)=e^{\int T(z)}+\Lambda^{2 N} e^{-\int T(z)}
$$

which we use to find $P_{\alpha}(z)$ from (2.47)

$$
P_{\alpha}(z)=w_{\alpha}(z)\left(e^{\int T(z)}-\Lambda^{2 N} e^{-\int T(z)}\right) .
$$

The constraints on $u_{k}$ and $w_{\alpha, k}$ come from imposing that the coefficients of the negative powers of $z$ in the Laurent series on the left hand side of (2.55) or (2.56) vanish. Since 
the coefficient of $z^{N-k}$ of (2.55) is linear in $u_{k}$ and does not depend on $u_{l}$ with $l>k$, setting the coefficient to zero gives a recursion relation for $u_{k}$ in terms of $u_{1}, u_{2}, \ldots, u_{k-1}$. We can solve the recursion relations to find $u_{k}$ as a polynomial in $u_{1}, \ldots, u_{N}$. Similarly, the coefficient of $z^{N-1-l}$ of (2.56) is linear in $w_{\alpha, k}$ and is independent of $w_{\alpha, l}$ with $l>k$. Hence, we get recursion relations for $w_{\alpha, k}$ with $k \geq N$ which determine $w_{\alpha, k}$ in terms of $w_{\alpha, 0}, \ldots, w_{\alpha, N-1}$ and $u_{1}, \ldots, u_{N}$.

We recast the formula $(2.49)$ as

$$
-\frac{P_{\alpha}(z) P^{\alpha}(z)}{2 P(z)}=\left(R(z)-W^{\prime}(z) / 2+\frac{c(z)}{2 T(z)}\right)\left(e^{\int T(z)}-\Lambda^{2 N} e^{-\int T(z)}\right) .
$$

We will not use this formula except for next subsection where we relate it to 2.55) and $(2.56)$.

\section{3. $U(1)_{\text {free }}$ and Shift Symmetry}

We decompose the $U(N)$ gauge symmetry as $S U(N) \times U(1)_{\text {free }}$ on the level of Lie algebras. We embed the $U(1)_{\text {free }}$ photino $\mathcal{W}_{\alpha}$ into the $U(N)$ gauge theory as $\mathcal{W}_{\alpha} \times 1_{N \times N}$. All fields are in the adjoint representation of the $U(N)$ gauge symmetry, hence they are neutral under the diagonal $U(1)_{\text {free }}$ which gets decoupled from the rest of the theory. It is described completely by the free $\mathcal{W}_{\alpha} \mathcal{W}^{\alpha}$ action. In the chiral ring, the $U(1)_{f}$ ree photino is described by an anticommuting number $\psi_{\alpha}$ because the chiral operator $\mathcal{W}_{\alpha}$ is independent of position. Hence, the $U(1)_{\text {free }}$ part of the gaugino generating functions are

$$
\begin{aligned}
w_{\alpha}(z) & =\frac{1}{4 \pi} \operatorname{Tr} \frac{\psi_{\alpha}}{z-\Phi}=\frac{1}{4 \pi} \psi_{\alpha} T(z) \\
R(z) & =-\frac{\psi_{\alpha}}{4 \pi} w^{\alpha}(z)-\frac{1}{32 \pi^{2}} \psi_{\alpha} \psi^{\alpha} T(z) .
\end{aligned}
$$

It follows from the decoupling of $U(1)_{\text {free }}$ that the theory has an exact symmetry $W_{\alpha} \rightarrow W_{\alpha}+4 \pi \psi_{\alpha} 1_{N \times N}$ that shifts $W_{\alpha}$ by an anticommuting $c$-number. This symmetry acts on the chiral operators by

$$
\begin{aligned}
\delta r_{k} & =-w_{\alpha, k} \psi^{\alpha}-\frac{1}{2} \psi_{\alpha} \psi^{\alpha} u_{k}, \\
\delta w_{\alpha, k} & =u_{k} \psi_{\alpha} \\
\delta u_{k} & =0
\end{aligned}
$$

We define the field $\tilde{W}_{\alpha}=W_{\alpha}+4 \pi \psi_{\alpha}$, then the shift symmetry is generated by $\partial / \partial \psi_{\alpha}$. Invariance under this symmetry implies that the chiral ring relations do not depend on $\psi_{\alpha}$ 
when they are expressed in terms of $\tilde{W}_{\alpha}$. We substitute $\tilde{W}_{\alpha}$ instead of $W_{\alpha}$ into the definitions (2.5), (2.16), (2.23) and (2.28) to find the shift symmetric resolvents and polynomials

$$
\begin{aligned}
\tilde{R}(z) & =R(z)-w_{\alpha}(z) \psi^{\alpha}-\frac{1}{2} \psi_{\alpha} \psi^{\alpha} T(z), \\
\tilde{f}(z) & =f(z)-4 \psi_{\alpha} \rho^{\alpha}(z)-2 \psi_{\alpha} \psi^{\alpha} c(z), \\
\tilde{w}_{\alpha}(z) & =w_{\alpha}(z)+\psi_{\alpha} T(z), \\
\tilde{P}_{\alpha}(z) & =P_{\alpha}(z)+\psi_{\alpha} P^{\prime}(z) .
\end{aligned}
$$

Finally, we can write down the shift invariant form of the anomaly relations 2.21)

$$
\tilde{R}^{2}(z)=W^{\prime}(z) \tilde{R}(z)+\frac{1}{4} \tilde{f}(z)
$$

and the shift invariant $\mathcal{N}=2$ equations

$$
\tilde{R}(z)=-\frac{\tilde{P}_{\alpha}(z) \tilde{P}^{\alpha}(z)}{2 P^{\prime}(z) \sqrt{P^{2}(z)-4 \Lambda^{2 N}}}+\frac{W^{\prime}(z)}{2}-c(z) \frac{\sqrt{P^{2}(z)-4 \Lambda^{2 N}}}{2 P^{\prime}(z)} .
$$

The second and the third term are independent of $\psi_{\alpha}$, whence they contribute only to the lowest component of $\tilde{R}(z)$ which is $R(z)$ itself. To get the relations for $\mathcal{N}=2$ gauge theory we set the superpotential to zero

$$
\tilde{R}(z)=-\frac{\tilde{P}_{\alpha}(z) \tilde{P}^{\alpha}(z)}{2 P^{\prime}(z) \sqrt{P^{2}(z)-4 \Lambda^{2 N}}} .
$$

The shift invariant $\mathcal{N}=2$ relation that combines the formula for $T(z)$ and $w_{\alpha}(z)$ is

$$
\tilde{w}_{\alpha}(z)=\frac{\tilde{P}_{\alpha}(z)}{\sqrt{P^{2}(z)-4 \Lambda^{2 N}}}
$$

This relation holds for any superpotential unlike 2.63) which is valid only for zero superpotential.

The equation (2.64) is the unique shift symmmetric completion of the $\mathcal{N}=2$ formula for $T(z)$. Each term in formula for $w_{\alpha}(z)$ depends on $W_{\alpha}$ and hence gives a nonzero contribution by shift symmetry to the formula for $T(z)$. Barring unexpected cancellations, the formula for $w_{\alpha}(z)$ is fixed by requiring that it shifts to the correct relation for $T(z)$. The formula for $R(z)$ is not fixed by shift symmetry from the formula for $w_{\alpha}(z)$. It can have additional terms that are independent of $W_{\alpha}$ which get shifted to zero, hence they are not constrained by the formula for $w_{\alpha}$. These terms are absent for the $\mathcal{N}=2$ gauge theory, 
where the formula (2.63) for $R(z)$ gives the response to nonzero vacuum expectation value of the $U(1)$ photinos hence it does not contain terms independent of $W_{\alpha}$. There are such terms when we turn on superpotential as is manifest from (2.62).

The shift invariant integral relation that combines $(2.55),(2.56)$ and $(2.57)$ is

$$
-\frac{\tilde{P}_{\alpha}(z) \tilde{P}^{\alpha}(z)}{2 P^{\prime}(z)}=\tilde{R}(z)\left(e^{\int T(z)}-\Lambda^{2 N} e^{-\int T(z)}\right) .
$$

In this form, the formula for $-P_{\alpha}(z) P^{\alpha}(z) / P^{\prime}(z) P(z)$ goes by shift symmetry into the formula for $P_{\alpha}(z)$ which goes to the first derivative of the formula for $P(z)$. The $\mathcal{N}=2$ relation for $P(z)$ and $P_{\alpha}(z)$ combines is

$$
\tilde{P}_{\alpha}(z)=\tilde{w}_{\alpha}(z)\left(e^{\int T(z)}-\Lambda^{2 N} e^{-\int T(z)}\right)
$$

Similarly to (2.64) this formula holds for any superpotential.

\section{Solution of the Chiral Ring}

\subsection{U(2) Gauge Theory with Cubic Superpotential}

Before giving a general proof that the chiral ring determines all the vacua of the theory we will illustrate this in detail in the case of the $U(2)$ gauge theory with cubic superpotential

$$
W^{\prime}(\Phi)=\frac{1}{3} \operatorname{Tr} \Phi^{3}-\frac{1}{2} \operatorname{Tr} \Phi^{2}
$$

We can always put a cubic superpotential into this simple form by rescaling and shifting $\Phi$ and $W_{\alpha}$. Let us count the number of chiral operators that we need to consider after taking into account the recursion relations for the moments. A $2 \times 2$ matrix $\Phi$ is described by two independent gauge invariant chiral operators $u_{1}$ and $u_{2}$ which determine the remaining $u_{i}$ 's from (2.38). There are two independent gaugino operators $w_{\alpha, 0}$ and $w_{\alpha, 1}$ which determine

the remaining $w_{\alpha, i}$ 's from (2.47). For cubic superpotential, the $r_{i}$ 's are determined by $r_{0}$ and $r_{1}$ from the first anomaly equation (2.21). Hence, we have already reduced the number of chiral operators that generate the ring down to six.

To get started, we solve for vacua in the classical case. We treat $u_{k}$ 's as numbers and ignore the nilpotents $w_{\alpha, k}$ and $r_{k}$. The superpotential has two critical points, $\lambda=$ 0,1 . Hence, the theory has three vacua corresponding to different arrangements of the eigenvalues of $\Phi$ among the critical points $\Phi=\operatorname{diag}(0,0), \operatorname{diag}(0,1), \operatorname{diag}(1,1)$. The vacuum 
$\Phi=\operatorname{diag}(1,0)$ is gauge equivalent to the vacuum $\Phi=\operatorname{diag}(0,1)$. The vacua are described by the gauge invariant operators

$$
u_{1}=u_{2}=u_{3}=\ldots=0,1,2 \text {. }
$$

These values of $u_{k}$ obey all chiral ring relations by definition. We will now show that these are no additional solutions to the chiral ring relations. We expand the equations for $\Phi$ (2.14) in $1 / z$ to get

$$
\operatorname{Tr} \Phi^{k} W^{\prime}(\Phi)=u_{k+2}-u_{k+1}=0
$$

Hence the equations of motion set all moments of $T(z)$ equal to $u_{1}$

$$
u_{k}=u_{1}
$$

giving us one dimensional family of solutions parametrized by $u_{1}$. However, we know from above that only three solutions of this family correspond to supersymmetric vacua of the theory. Hence, the relations coming from the equations of motions are not restrictive enough. Fortunately, $u_{3}, u_{4}, \ldots$ are determined by $u_{1}, u_{2}$ from (2.38), so we have additional constraints which we can impose on the above one dimensional family of solutions. Substituting (3.4) into the relation (A.7) $u_{3}=-\frac{1}{2} u_{1}^{3}+\frac{3}{2} u_{1} u_{2}$, we find

$$
u_{1}\left(u_{1}-1\right)\left(u_{1}-2\right)=0
$$

The solutions of this equation are $u_{1}=0,1,2$ which are the expectation values of $u_{1}$ in the three supersymmetric vacua discussed above. The idempotents corresponding to these vacua are $\frac{1}{2}\left(u_{1}-1\right)\left(u_{1}-2\right),-u_{1}\left(u_{1}-2\right), \frac{1}{2} u_{1}\left(u_{1}-1\right)$. Each solution of the chiral ring corresponds to a supersymmetric vacuum of the gauge theory.

The calculation in the quantum case is similar except that we need to keep track of $r_{k}$ 's which get a nonzero expectation value from gaugino condensation. We will take into account the infinitensimal $w_{\alpha}(z)$ to find the low energy gauge group. We take the last anomaly equation (2.21)

$$
2 R(z) T(z)=\operatorname{Tr} \frac{W^{\prime}(\Phi)}{z-\Phi}
$$

and expand it in $1 / z$ to find the recursion relations for $u_{k}$

$$
u_{k+2}=u_{k+1}+2 \sum_{i=1}^{k} u_{i} r_{k-i}
$$


We compare these with the equations (A.7) for $u_{3}$ and $u_{4}$ in terms of $u_{1}$ and $u_{2}$. This allows us to express $u_{2}, r_{0}$ and $r_{1}$ in terms of $u_{1}$

$$
\begin{aligned}
& u_{2}=u_{1} \\
& r_{0}=-\frac{1}{8} u_{1}\left(u_{1}-1\right)\left(u_{1}-2\right) \\
& r_{1}=-\frac{1}{16} u_{1}^{2}\left(u_{1}-1\right)\left(u_{1}-2\right)+\Lambda^{4} .
\end{aligned}
$$

The constraint for $u_{1}$ comes from comparing the two formulas for $u_{5}$. We use that $r_{2}=$ $r_{1}+r_{0}^{2}$, which comes from the $1 / z^{2}$ term of the first equation (2.21)

$$
R^{2}(z)=-\frac{1}{32 \pi^{2}} \operatorname{Tr} W_{\alpha} W^{\alpha} \frac{W^{\prime}(\Phi)}{z-\Phi} .
$$

We have

$$
\left(u_{1}-1\right)\left[u_{1}^{2}\left(u_{1}-2\right)^{2}-64 \Lambda^{4}\right]=0
$$

which determines the location of the roots of the chiral ring relations in the complex $u_{1}$ plane. The equation (3.8) has five roots for $u_{1}$.

Quantum corrections do not move the vacuum at $u_{1}=1$ from the classical position in $\mathcal{N}=2$ moduli space because all monopoles are massive and the instanton corrections to the moduli space move the classical vacua only for superpotential of degree five or higher. The vacuum has zero total gaugino condensate (3.8)

$$
S=S_{1}+S_{2}=0
$$

Instantons generate gaugino condensation in each of the $U(1)$ factors leading to $r_{1}=\Lambda^{4}$. There are two vacua with $u_{1}=1+\sqrt{1 \pm 8 \Lambda^{2}}$ near the classical critical point $\Phi_{c l}=\operatorname{diag}(1,1)$ from the strongly coupled $S U(2)$ and two more vacua with $u_{1}=1-\sqrt{1 \pm 8 \Lambda^{2}}$ near $\Phi_{c l}=\operatorname{diag}(0,0)$. The vacua have nonzero gaugino condensation $r_{0}= \pm \sqrt{1 \pm 8 \Lambda^{2}}$.

To find the rank of the low energy gauge group we solve the linear equations for $w_{\alpha, k}$ and count the dimension of the space of solutions. We will justify this prescription in the next section. The gaugino operators $w_{\alpha, 0}$ and $w_{\alpha, 1}$ obey relations that are generated by expanding (2.47) in powers of $1 / z$. This gives a single constraint

$$
\left(u_{1}-1\right)\left(u_{1} w_{\alpha, 0}-2 w_{\alpha, 1}\right)=0
$$

At the vacuum with $u_{1}=1$, the constraint becomes trivial hence $w_{\alpha, 0}$ and $w_{\alpha, 1}$ are independent. The vacuum has $U(1)^{2}$ low energy gauge symmetry. The vacua with $u_{1} \neq 1$ have only one independent photino because (3.12) has a one dimensional family of solutions $w_{\alpha, 1}=\frac{u_{1}}{2} w_{\alpha, 0}$. Hence these vacua have $U(1)$ low energy gauge group. 


\subsection{Classical Case}

We will now show that the supersymmetric vacua are in one to one correspondence with the solutions of the chiral ring relations. We will warm up on the classical case.

We have found two different formulas for the resolvents in terms of the first $n$ or $N$ moments. Comparing the formulas for resolvents from (2.17) with (2.25), (2.30) and (2.31) we obtain nontrivial relations for the first $\max (N, n)$ moments

$$
\begin{aligned}
T(z) & =\frac{c(z)}{W^{\prime}(z)}=\frac{P^{\prime}(z)}{P(z)}, \\
w_{\alpha}(z) & =\frac{\rho_{\alpha}(z)}{W^{\prime}(z)}=\frac{P_{\alpha}(z)}{P(z)}, \\
R(z) & =-\frac{f(z)}{4 W^{\prime}(z)}=\frac{P_{R}(z)}{P(z)}
\end{aligned}
$$

Expanding these equations in $1 / z$ we would get an infinite number of equations for the moments. Instead, we rewrite the equations as

$$
\begin{aligned}
P^{\prime}(z) W^{\prime}(z) & =P(z) c(z), \\
P_{\alpha}(z) W^{\prime}(z) & =P(z) \rho_{\alpha}, \\
P_{R}(z) W^{\prime}(z) & =-\frac{1}{4} P(z) f(z)
\end{aligned} .
$$

Then expanding in $z$ we get a finite number of chiral relations to solve. Assume that the superpotential $W^{\prime}(z)=\prod_{i=1}^{n}\left(z-\lambda_{i}\right)$ has $n$ distinct critical points. The most general solution of (3.14) can be expressed in terms of auxiliary polynomials $F(z), H(z), Q(z)$ and $\tilde{c}(z)$

$$
\begin{gathered}
W^{\prime}(z)=Q(z) F(z), \\
c(z)=Q(z) \tilde{c}(z), \\
P(z)=H(z) F(z), \\
P^{\prime}(z)=H(z) \tilde{c}(z),
\end{gathered}
$$

where $F(z)=\prod_{i=1}^{k}\left(z-\lambda_{i}\right)$ is a polynomial of degree $k . F(z)$ has only single roots. They are the $k$ occupied critical points of the superpotential. The resolvent $T(z)$ is (3.13)

$$
T(z)=\frac{\tilde{c}(z)}{F(z)}=\sum_{j=1}^{k} \frac{N_{j}}{z-\lambda_{j}}
$$

The second equality holds because the polynomial $\tilde{c}(z)$ of degree $k-1$ can be expressed as a linear combination of the $k$ linearly independent polynomials $F_{i}(z)=\prod_{j \neq i}\left(z-\lambda_{j}\right)$. 
The $N_{i}$ 's are integers being the logarithmic residues of $P(z)$. They give the multiplicity of the eigenvalue $\lambda_{i}$ in $\Phi$. The solution is completely specified by $N_{i}$ 's. It corresponds to the vacuum in which $\Phi$ breaks the $U(N)$ gauge symmetry to $U\left(N_{1}\right) \times U\left(N_{2}\right) \times \ldots \times U\left(N_{k}\right)$. Taking different $N_{i}$ gives all vacua of the gauge theory. The expectation values of $u_{k}$ 's in a particular vacuum are generated by $T(z)$ of the corresponding solution of the chiral ring relations. The roots of the chiral ring relations are in one to one correspondence with the vacua of the theory.

The equations (3.14) linear in $W_{\alpha}$ determine the number of unbroken $U(1)$ 's. Their general solution

$$
\begin{aligned}
& P_{\alpha}(z)=H(z) \sigma_{\alpha}(z), \\
& \rho_{\alpha}(z)=Q(z) \sigma_{\alpha}(z)
\end{aligned}
$$

is written in terms of the arbitrary polynomial $\sigma_{\alpha}(z)$ of degree $k-1$ which has $k$ independent anticommuting coefficients

$$
w_{\alpha}(z)=\frac{\sigma_{\alpha}(z)}{F(z)} .
$$

Hence the vacuum has $k U(1)$ gauginos coming from the $U(1)$ factors of $U\left(N_{i}\right)$

$$
\hat{w}_{\alpha, i}=\frac{1}{4 \pi} \operatorname{Tr} W_{\alpha} P_{i}
$$

where $P_{i}$ is the projector on the subspace $\Phi=\lambda_{i}$ preserved by the $U\left(N_{i}\right)$ gauge symmetry. We use a hat to distinguish $\hat{w}_{\alpha i}$ from $w_{\alpha, i}$ Similarly, $R(z)$ is given in terms of an arbitrary polynomial $q(z)$ of degree $k-1$

$$
R(z)=\frac{q(z)}{F(z)}
$$

which indicates that the vacuum has $k$ independent $r_{i}$ 's. Linear combinations of $r_{i}$ give the gaugino bilinears

$$
S_{i}=\frac{-1}{32 \pi^{2}} \operatorname{Tr} W_{\alpha} W^{\alpha} P_{i}
$$

of the $U\left(N_{i}\right)$ subgroup.

\subsection{Quantum Case}

The solution of the quantum case is similar to the classical case. We compare the perturbative formulas (2.22) for the resolvents $T(z)$ and $w_{\alpha}(z)$ with the nonperturbative formulas (2.38) and (2.47). We find the chiral ring relations

$$
\begin{gathered}
T(z)=\frac{c(z)}{\sqrt{W^{\prime 2}(z)+f(z)}}=\frac{P^{\prime}(z)}{\sqrt{P^{2}(z)-4 \Lambda^{2 N}}}, \\
w_{\alpha}(z)=\frac{\rho_{\alpha}(z)}{\sqrt{W^{\prime 2}(z)+f(z)}}=\frac{P_{\alpha}(z)}{\sqrt{P^{2}(z)-4 \Lambda^{2 N}}} .
\end{gathered}
$$


Expanding both sides of (3.22) in $1 / z$ and comparing the coefficients of the two Laurent series we obtain an infinite number of relations for the first $\max (n, N)$ moments of the resolvents.

We rewrite $(3.22)$ as

$$
\begin{aligned}
P^{\prime 2}(z)\left(W^{\prime 2}(z)+f(z)\right) & =\left(P^{2}(z)-4 \Lambda^{2 N}\right) c^{2}(z) \\
P_{\alpha}(z) c(z) & =P^{\prime}(z) \rho_{\alpha}(z)
\end{aligned}
$$

to get a finite number of equations. We have eliminated the square roots in the second equation using the first equation. Let us focus now on the first equation in (3.23). Expanding the equation in $z$ and comparing the coefficients we obtain a finite number of chiral ring relations that can be solved to find expectation values $u_{1}, \ldots, u_{\max (N, n)}, r_{0}, \ldots, r_{n-1}$ in all vacua. We obtain $2 N+2 n-1$ equations while the number of independent variables is $\max (N, n)+n$. Generically, the number of independent equations is larger than the number of variables.

To solve the quantum chiral ring relations, assume that the matrix curve $y_{m}^{2}=F_{2 g}(z)$ has genus $g$. Hence, the $\mathcal{N}=2$ curve has $N-g$ double roots

$$
P^{2}(z)-4 \Lambda^{2 N}=H_{N-g}^{2}(z) F_{2 g}(z)
$$

Taking derivative of (3.24) we find that $P^{\prime}(z)=H_{N-g}(z) \tilde{c}_{g-1}(z)$ is divisible by $H_{N-g}(z)$. To match single roots on both sides of (3.23) we must have

$$
W^{\prime 2}(z)+f(z)=Q_{n-g}^{2}(z) F_{2 g}(z)
$$

hence $c(z)=Q_{n-g} \tilde{c}_{g-1}(z)$. The equation (3.25) is the generalized condition for finding vacua for arbitrary degree of the superpotential [5]. We remark that even though the relation (3.25) has a direct physical interpretation in terms of condensation of $N-g$ massless monopoles and factorization of the matrix model curve, it is not a chiral ring relation because it does not hold in all vacua of the theory. The equations (3.23) are chiral ring operator statements valid in every vacuum of the gauge theory. Substituting the solution (3.25) into (3.22) we get the relation for $T(z)$ in terms of the matrix model curve

$$
T(z)=\frac{\tilde{c}(z)}{\sqrt{F_{2 g}(z)}} .
$$

To find the position of the supersymmetric vacua in the $\Phi$ moduli space we have set to zero the $U(1)$ photinos. We were allowed to do this because the expectation value of 
the photinos moves the vacua by an infinitensimaly small amount because of the nilpotent nature of the photino operators $w_{\alpha, i}$.

Substituting $c(z)$ and $P^{\prime}(z)$ into $(3.23)$ gives

$$
P_{\alpha}(z) Q_{n-g}(z)=H_{N-g}(z) \rho_{\alpha}(z)
$$

The general solution of this equation is

$$
\begin{aligned}
& P_{\alpha}(z)=H_{N-g}(z) \sigma_{\alpha, g-1}(z) \\
& \rho_{\alpha}(z)=Q_{n-g}(z) \sigma_{\alpha, g-1}(z)
\end{aligned}
$$

where $\sigma_{\alpha, g-1}(z)$ in an arbitrary polynomial of $g-1$ 'st degree. So $w_{\alpha}(z)$ is determined by the $g$ independent fermionic coefficients of $\sigma_{\alpha, g-1}(z)$

$$
w_{\alpha}(z)=\frac{\sigma_{\alpha, g-1}(z)}{\sqrt{F_{2 g}(z)}} .
$$

Along these directions, the photinos can take vacuum expectation values. Hence, each massive vacuum has massless fermionic moduli directions parametrized by the magnitude of the photino condensate. The photons that are supersymmetric partners of the massless photinos are massless as well. These are the freely propagating photons of the low energy effective gauge group. Hence, the number of $U(1)$ photons is equal to the number of massless photinos which is equal to the dimension of the fermionic moduli space. To find the dimension, it is enough to consider equations linear in $w_{\alpha}(z)$ and count the number of parameters describing their solution. This justifies the above calculation and implies that the vacuum corresponding to genus $g$ matrix model curve have $U(1)^{g}$ low energy gauge symmetry.

\subsection{Perturbative Chiral Ring}

Finally, let us consider the chiral ring that incorporates the perturbative corrections only. We turn off the nonperturbative corrections by setting the strong coupling scale $\Lambda$ to zero in chiral ring relations. The ideal of relations is generated by (3.23)

$$
P^{\prime 2}(z)\left(W^{\prime 2}(z)+f(z)\right)=P^{2}(z) c^{2}(z)
$$

and

$$
P_{\alpha}(z) c(z)=P^{\prime}(z) \rho_{\alpha}(z)
$$


As a simple consequence of (3.30), we observe that $\langle f(z)\rangle=0$ in every vacuum, because $W^{\prime 2}(z)+f(z)$ is a square of a polynomial if and only if $f(z)=0$ or $\operatorname{deg} f(z) \geq \operatorname{deg} W^{\prime}(z)$, but $f(z)$ has degree one smaller than $W^{\prime}(z)$. So we see from (2.22) that

$$
\langle R(z)\rangle=0,
$$

the gaugino condensate is vanishes to all orders in perturbation theory. The $r_{k}$ 's are nilpotent operators of the perturbative chiral ring because $\left\langle r_{k}\right\rangle=0$ for each solution of the perturbative relations (3.30). The nilpotency follows from Hilbert's Nullstellensatz, p. 412 of [6], which states that if a polynomial $g$ vanishes at every solution of an ideal $\mathcal{I}$ of polynomial relations then $g^{k}$ for sufficiently large $k$ is an element of the ideal $\mathcal{I}$. We will discuss in section on gaugino condensation that the actual nilpotency condition on $r_{k}$ is that the product of any $N r_{k}$ 's is zero in the perturbative chiral ring.

To find the positions of the vacua in the $\Phi$ moduli space, we set $f(z)$ to zero in (3.30). The chiral ring reduces to the classical chiral ring. Hence, the perturbative corrections do not shift the positions of the vacua in the $\Phi$ moduli space and the equations (3.31) give the correct number of $U(1)$ gauge symmetries. To account for the correct multiplicity of the vacua we need to retain the nilpotent $f(z)$. The multiplicity of the root equals the multiplicity of the supersymmetric vacua. The multiple root splits into single roots and the supersymmetric vacua separate in the $\Phi$ moduli space when we make $\Lambda$ nonzero.

In the example the chiral ring of $U(2)$ gauge theory with cubic superpotential, the classical ring is $u_{1}\left(u_{1}-1\right)\left(u_{1}-2\right)=0$ while the perturbative ring is

$$
u_{1}^{2}\left(u_{1}-1\right)\left(u_{1}-2\right)^{2}=0
$$

which can be obtained from (3.10) by setting $\Lambda=0$. The double roots correspond to the pairs of vacua that come from the strongly coupled $S U(2)$ vacua.

\section{Intersection of Vacua}

Generically, all vacua are located at different points in the $\Phi$ moduli space. By tuning the superpotential, we can make two or more vacua intersect. We will consider only the intersections at which mutually local monopoles are massless. The chiral ring relations will have a multiple root. Its multiplicity equals to the number of intersecting vacua. We notice that $R(z)$ is determined by the location of the vacua in the $N=2$ moduli space 
determines for given superpotential from (2.49). Hence, the intersecting vacua have the same expectation value of the moments of gaugino condensate.

Let us investigate the the low energy gauge group of the intersecting vacua. $T(z)$ determines the linear constrains (2.56) for $w_{\alpha}(z)$. So the rank of the low energy gauge group depends only on the position of the vacuum in the $\Phi$ moduli space. The intersecting vacua have the same low energy gauge group $U^{g}(1)$ with $g \geq g_{i}$ where $U(1)^{g_{i}}$ is the the gauge group of $i$ 'th vacuum near the intersection. The lower bound of the rank of the gauge group follows, because when tuning the superpotential to make the vacua intersect, the dimension of the space solution to (2.56) can suddenly jump up as some of the constraints for $w_{\alpha, k}$ become satisfied on a submanifold of the $\Phi$ moduli space.

Physically, the increase in the rank of the gauge group is connected with vanishing of condensate of monopoles at the vacuum. As the vacua approach each other, the dual Meissner effects of the confined $U(1)$ 's turns off. At the intersection the monopole has zero expectation value and the dual electric $U(1)$ is free. We will investigate monopole condensation using the low energy effective lagrangian [7,8] that includes the monopole fields

$$
\mathcal{L}_{\text {eff }}=\sum_{k=0}^{n} \frac{g_{k}}{k+1} \operatorname{Tr} \Phi^{k+1}+\sum_{i=1}^{N} M_{i}(\Phi) m_{i} \tilde{m}_{i} .
$$

The mass of the $i$ 'th monopole $M_{i}(\Phi)$ is a function on the $\mathcal{N}=2$ moduli space. We can find the monopole condensate by varying these equations with respect to $u_{1}, \ldots u_{N}, m_{1}, \ldots, m_{N}$ and $\tilde{m}_{1}, \ldots, \tilde{m}_{N}$. For present purposes it is enough to notice that the monopole condensates depends continuously on the superpotential and the $u_{i}$ 's. Thus, the monopole condensate associated with the deconfining $U(1)$ turns off continuously on the approach of the intersection. This follows from the formula (3.16) of [9] for the value of monopole condensates.

We will now illustrate this behavior for $U(2)$ gauge theory with the cubic superpotential $W^{\prime}(z)=z^{2}-z$ which we analyzed in previous section. When

$$
8 \Lambda^{2}=1
$$

the two vacua at $u_{1}=1 \pm \sqrt{1-8 \Lambda^{2}}$ intersect with the $u_{1}=1$ vacuum. Ignoring the photinos for a moment we see that the chiral ring is generated by $u_{1}$, which obeys the constraint (3.10)

$$
\left(u_{1}-1\right)^{3}\left(u_{1}^{2}-2 u_{1}-1\right)=0 .
$$

The equation (4.3) has a triple root at $u_{1}=1$. The local ring at the triple root is three dimensional. The basis elements behave as $1,\left(u_{1}-1\right),\left(u_{1}-1\right)^{2}$ near the root and vanish 
at the two other roots of (4.3). To find the expectation value of a chiral operator in the intersecting vacua, we expand it in the local ring and read off the coefficient at the idempotent element.

We see from (3.12) that the gauge group of each of them gets enlarged to $U(1)^{2}$. The rank of the gauge group depends only on the $\Phi$ moduli space. We can see the increase in the rank of the gauge group directly from the low energy effective action of the theory near the intersection points (4.1)

$$
W(\Phi)=\frac{u_{3}}{3}-\frac{u_{2}}{2}+m\left(2 u_{2}-u_{1}^{2} \pm \Lambda^{2}\right) q \tilde{q}
$$

The monopole condensate in the vacua with $U(1)_{\text {free }}$ gauge symmetry is

$$
q \tilde{q}=m^{\prime}\left(u_{1}-1\right)
$$

where $m^{\prime}$ is a constant. Near $u_{1}=1$, the condensate which confines the second $U(1)$ goes to zero and the dual Meissner effect continuously turns off.

\section{Gaugino Condensation}

We have seen that chiral ring gives all the supersymmetric vacua together with the expectation values of all chiral operators. The chiral ring can be used to extract general statements about the properties of the vacua. We have seen one example of this when we showed that chiral ring encodes the low energy group of the vacua. The dimension of the gauge group was shown to be equal to the dimension of the of the fermionic moduli parametrizing the condensate of the $U(1)$ photinos. We will now analyze the chiral ring relations satisfied by the gaugino bilinears $r_{i}$ and their implications for gaugino condensation. For simplicity, we will assume throughout this section that the photino expectation values vanish.

\subsection{Classical case}

Classically, $W_{\alpha}$ is an $N \times N$ matrix of two component grassmanian numbers. The operators $r_{i} \sim \operatorname{Tr} \Phi^{i} W_{\alpha} W^{\alpha}$ are bosonic operators constructed from fermionic operators. These operators are nilpotent because of the anticommutativity of $W_{\alpha}$. We have

$$
r_{i_{1}} \ldots r_{i_{N^{2}+1}}=0
$$


because $W_{\alpha}$ consists of $N^{2}$ two component fermions. In the chiral ring, the relations

$$
\begin{array}{r}
\left\{W_{\alpha}, W_{\beta}\right\}=0 \\
{\left[W_{\alpha}, \Phi\right]=0}
\end{array}
$$

imply a more powerful result. These identities generate the ideal $\mathcal{I}$ which is the subideal of the full ideal of classical relations. The remaining the classical relations have been discussed in the section 2. We denote $W_{1}$ and $W_{2}$ by $A$ and $B$. Then the ideal $\mathcal{I}$ is generated by $A^{2}, B^{2}$ with both $A$ and $B$ commuting with $\Phi$ and anticommuting with each other. For example the authors of [3] showed that

$$
r_{0}^{N}=S^{N}=0
$$

holds in the chiral ring of the pure $U(N)$ gauge theory. This relation continues to be valid when we add the adjoint field $\Phi$ because

$$
r_{0}=-\frac{1}{32 \pi^{2}} \operatorname{Tr} W_{\alpha} W^{\alpha}=-\frac{1}{16 \pi^{2}} \operatorname{Tr} A B
$$

does not depend on $\Phi$, so the proof from [3] for the pure $U(N)$ gauge theory is still valid. There is a similar relation for the product of arbitrary $N$ moments of $R(z)$

$$
r_{k_{1}} r_{k_{2}} \ldots r_{k_{N}}=0
$$

To derive (5.5), we closely follow [10]. We construct the tensor $F(A)$ from $A$

$$
F^{i_{1} i_{2} \ldots i_{N}}(A)=\epsilon^{j_{1} j_{2} \ldots j_{N}} A_{j_{1}}^{i_{1}} A_{j_{2}}^{i_{2}} \ldots A_{j_{N}}^{i_{N}}
$$

The epsilon tensor on the right hand side picks out the completely antisymmetric part in the $j$ indices of $A$, hence by anticommutativity of $A, F$ is completely symmetric in the $i$ indices. We will show later that $F(A)$ is contained in the ideal $\mathcal{I}$. We also define a complementary tensor from $B$ and $\Phi$

$$
G_{i_{1} i_{2} \ldots i_{N}}(B)=\left(-\frac{1}{16 \pi^{2}}\right)^{N} \epsilon_{l_{1} l_{2} \ldots l_{N}}\left(\Phi^{k_{1}} B\right)_{i_{1}}^{l_{1}}\left(\Phi^{k_{2}} B\right)_{i_{2}}^{l_{2}} \ldots\left(\Phi^{k_{N}} B\right)_{i_{N}}^{l_{N}} .
$$

Since $F(A)$ is contained in the ideal $\mathcal{I}$, so is its contraction with $G(B)$

$$
F(A) \cdot G(B)=\epsilon^{i_{1} i_{2} \ldots i_{N}} A_{j_{1}}^{i_{1}} A_{j_{2}}^{i_{2}} \ldots A_{j_{N}}^{i_{N}} \epsilon_{l_{1} l_{2} \ldots l_{N}}\left(\Phi^{k_{1}} B\right)_{i_{1}}^{l_{1}}\left(\Phi^{k_{2}} B\right)_{i_{2}}^{l_{2}} \ldots\left(\Phi^{k_{N}} B\right)_{i_{N}}^{l_{N}}
$$


We arrange the right hand side of (5.8) using the identity

$$
\epsilon^{j_{1} j_{2} \ldots j_{N}} \epsilon_{l_{1} l_{2} \ldots l_{N}}=\delta_{l_{1}}^{j_{1}} \delta_{l_{2}}^{j_{2}} \ldots \delta_{l_{N}}^{j_{N}} \pm \text { permutations. }
$$

The delta tensors contract the indices between $F(A)$ and $G(B)$ making $F(A) \cdot G(B)$ into a sum of terms

$$
\operatorname{Tr} \Phi^{p_{1}}(A B)^{s_{1}} \operatorname{Tr} \Phi^{p_{2}}(A B)^{s_{2}} \ldots \operatorname{Tr} \Phi^{p_{N}}(A B)^{s_{N}}
$$

with various $p_{i}$ and $s_{i}$. In writing (5.10) we used the fact that $\Phi$ commutes with $A$ and $B$ to collect $\Phi$ 's to the left of each trace. The term coming from the trivial permutation in (5.9) is

$$
r_{k_{1}} r_{k_{2}} \ldots r_{k_{N}}=\left(-\frac{1}{16 \pi^{2}}\right)^{N} \operatorname{Tr} \Phi^{k_{1}} A B \operatorname{Tr} \Phi^{k_{2}} A B \ldots \operatorname{Tr} \Phi^{k_{N}} A B .
$$

The remaining permutations give terms with some $s_{i}>1$ hence they are contained in the ideal $\mathcal{I}$.

To complete the proof, we will show that $F(A)$ is in the ideal $\mathcal{I}$. Since $F(A)^{i_{1} i_{2} \ldots i_{N}}$ is symmetric in its indices, we can set them to the same value. We will show that

$$
F(A)^{N N \ldots N}=\epsilon^{j_{1} j_{2} \ldots j_{N}} A_{j_{1}}^{N} A_{j_{2}}^{N} \ldots A_{j_{N}}^{N}
$$

is proportional to

$$
\epsilon^{N j_{1} j_{2} \ldots j_{N}}\left(A^{2}\right)_{j_{1}}^{N} A_{j_{2}}^{N} \ldots A_{j_{N-1}}^{N}
$$

which is in the ideal $\mathcal{I}$ because it is a multiple of $A^{2}$. We can write (5.13) as

$$
\sum_{x=1}^{N} \epsilon^{N j_{1} j_{2} \ldots j_{N-1}} A_{x}^{N} A_{j_{1}}^{x} A_{j_{2}}^{N} \ldots A_{j_{N-1}}^{N} .
$$

The expression

$$
A_{x}^{N} A_{j_{1}}^{N} A_{j_{2}}^{N} \ldots A_{N-1}^{N}
$$

is antisymmetric in its $N-1$ indices $x, j_{1}, j_{2}, \ldots, j_{N-1}$, hence it is a nonzero multiple of

$$
\epsilon_{x j_{2} j_{3} \ldots j_{N-1} k} \epsilon^{k l_{1} l_{2} \ldots l_{N-1}} A_{l_{1}}^{N} A_{l_{2}}^{N} \ldots A_{l_{N-1}}^{N} .
$$

We substitute this into (5.14)

$$
\sum_{x=1}^{N} \epsilon^{N j_{1} j_{2} \ldots j_{N-1}} \epsilon_{x j_{2} j_{3} \ldots j_{N-1} k} \epsilon^{k l_{1} l_{2} \ldots l_{N-1}} A_{j_{1}}^{x} A_{l_{1}}^{N} A_{l_{2}}^{N} \ldots A_{l_{N-1}}^{N}
$$

and use (5.9) to express the product of the first two epsilon tensors as a multiple of $\delta_{x}^{N} \delta_{k}^{j_{1}}-\delta_{k}^{N} \delta_{x}^{j_{1}}$. We find that (5.14) is a nonzero multiple of

$$
\left(\delta_{x}^{N} \delta_{k}^{j_{1}}-\delta_{k}^{N} \delta_{x}^{j_{1}}\right) \epsilon^{k l_{1} l_{2} \ldots l_{N-1}} A_{j_{1}}^{x} A_{l_{1}}^{N} A_{l_{2}}^{N} \ldots A_{l_{N-1}}^{N} .
$$

The term contracted with $\delta_{k}^{N} \delta_{x}^{j_{1}}$ are proportional to the $U(1)$ photino $\operatorname{Tr} A$ which we took to be zero. The term contracted with $\delta_{x}^{N} \delta_{k}^{j_{1}}$ is $F^{N N \ldots N}(A)$ as promised. 


\subsection{Quantum case}

Quantum mechanically, all vacua of the theory have nonzero gaugino condensation. This follows because all solutions of the equation (3.23)

$$
P^{\prime 2}(z)\left(W^{\prime 2}(z)+f(z)\right)=\left(P^{2}(z)-4 \Lambda^{2 N}\right) c^{2}(z)
$$

have $f(z) \neq 0$. We must have $f(z) \neq 0$ to insure that the left hand side of (5.19) is not a square of a polynomial, since the right hand side is cannot be written as a square of a polynomial when $\Lambda \neq 0$. Nonzero $f(z)$ is equivalent to nonzero gaugino condensation which can be seen from the equation $(2.22)$

$$
R(z)=\frac{1}{2}\left(W^{\prime}(z)-\sqrt{W^{\prime 2}(z)+f(z)}\right)
$$

for the generating function $R(z)$.

For a generic shape of the superpotential, we expect that the coefficients of the polynomial $f(z)$ are generic and nonzero. Hence, generically, all the moments $r_{k}$ are nonzero. In a particular vacuum, the first few moments can vanish if the gaugino condensates $S_{i}$ of the $U\left(N_{i}\right)$ subgroups cancel among each other when added up to make the gauge invariant operators

$$
r_{0} \sim \sum S_{i}, r_{1} \sim \sum \lambda_{i} S_{i}, \ldots
$$

In this case, some of the higher traces $r_{k}$ must be nonzero. Actually, infinitely many moments $r_{k}$ do not vanish. This follows from the fact that expanding the square root in (5.20) in powers of $1 / z$ we obtain Laurent series with infinite number of nonzero terms.

We would like to find the quantum version of the classical formulae (5.5). The product of $N$ gaugino bilinears is generated by instantons. We expect the $l$ instanton contribution to be proportional to the exponential of the $l$ instanton action $e^{-l S_{\text {inst }}}=\Lambda^{2 l N}$. The zero instanton term is absent. This expresses the absence of perturbative contribution to the gaugino condensation (3.32). The coefficient in front of the exponential is a polynomial in $u_{k}$ because the expectation value of the gaugino condensate depends on the position of the vacuum in the $\Phi$ moduli space. In summary, nonperturbative effects correct (5.5) to

$$
r_{k_{1}} r_{k_{2}} \ldots r_{k_{N}}=\sum_{l>0} \Lambda^{2 l N} Q_{l, k_{1} k_{2} \ldots k_{N}}\left(u_{1}, u_{2}, \ldots, u_{N}\right)
$$

The dimension of the left hand side is $3 N+\sum_{i} k_{i}$ hence the dimension of the polynomial $Q_{l, k_{1} k_{2} \ldots k_{N}}$ is $(3-2 l) N+\sum_{i} k_{i}$. Recalling that the dimension of $u_{k}$ and $\Lambda^{2 N}$ is $k$ and 
$2 N$ respectively, dimensional analysis gives us a simple constraint on $Q$. For example, $r_{0}^{N}=S^{N}$ can have only one instanton contribution, since $Q_{l, 00 \ldots 0}$ for $l>1$ would have negative dimension $-(l-1)$, which is a contradiction. $Q$, being a polynomial in $u_{k}$, has always positive dimension. The general form of $Q_{l, k_{1} k_{2} \ldots k_{N}}\left(u_{1}, u_{2}, \ldots, u_{N}\right)$ is not know. It is a complicated polynomial in $u_{i}$ that depends on the superpotential in a nontrivial way. Also, $Q_{l}$ 's are not uniquely defined. The chiral ring has often relations that express a polynomial in $u_{k}$ as $\Lambda^{2 N}$ times another polynomial of dimension $2 N$ less than the original polynomial. Adding $\Lambda^{2 l N}$ times this relation to the right hand side of (5.22) we change $Q_{l}$ and $Q_{l+1}$ without affecting the total sum. This is related to the fact that $\Lambda^{2 N}$ has the same quantum numbers as $\Phi^{2 N}$.

For the example of $U(2)$ gauge theory with cubic superpotential from section (3.1), the formulas for the product of two $r_{0}$ and $r_{1}$ are

$$
\begin{aligned}
r_{0}^{2} & =\left(u_{1}-1\right)^{2} \Lambda^{4} \\
r_{0} r_{1} & =\frac{\Lambda^{4}}{8} u_{1}\left(u_{1}-1\right)\left(3 u_{1}-2\right) \\
r_{1}^{2} & =\frac{\Lambda^{4}}{8} u_{1}^{3}\left(u_{1}-1\right)+\Lambda^{8} .
\end{aligned}
$$

We obtained these by multiplying the formulas (3.8) that express $r_{0}$ and $r_{1}$ in terms of $u_{1}$. To get the overall $\Lambda^{4}$ factor we have used the quintic equation (3.10) for $u_{1}$. We see that $r_{1}^{2}$ has also a two instanton contribution proportional to $\Lambda^{8}$. The product of any two moments $r_{i}$ and $r_{j}$ can be easily worked out from (5.23) because the higher moments can expressed as polynomials in $r_{0}$ and $r_{1}$ with the help of recursion relations obtained by expanding (3.9) in $1 / z$

$$
r_{k+2}=r_{k+1}+\sum_{i=0}^{k-1} r_{i} r_{k-i-1} .
$$

We find that the product can be written as a sum of terms that are polynomials of degree two or higher in $r_{0}$ and $r_{1}$. We rewrite these polynomials with the $\Lambda^{4 l}$ prefactor using (5.23)

$$
r_{i} r_{j}=\sum_{l>0} \Lambda^{4 l} Q_{l, i j}\left(u_{1}\right)
$$

\section{Examples}

In this section we give additional examples to illustrate in detail how the chiral ring determines the vacua of the gauge theory. 


\subsection{Unbroken Gauge Group}

In our first example we consider the $U(N)$ gauge theory with unbroken gauge group. For simplicity we will assume that the superpotential has one critical point $W(\Phi)=\frac{1}{2} m \Phi^{2}$. The theory with quadratic superpotential for the adjoint field was solved first by Douglas and Shenker [11]. Semiclassically, $\Phi$ is a massive scalar field with zero expectation value preserving the $U(N)$ gauge symmetry. The $S U(N)$ subgroup of the $U(N)$ gauge group gets strongly coupled by nonperturbative effects and the low energy gauge group is the decoupled $U(1)_{\text {free. }}$ There are $N$ strongly couples massive confining vacua with nonzero gaugino condensation. They are symmetrically distributed around the origin of the $S$ plane.

We will now study the full chiral ring of the gauge theory keeping both linear and quadratic terms in $w_{\alpha}(z)$. We substitute $c(z)=m N, f(z)=-4 m S$ and $\rho_{\alpha}(z)=m w_{\alpha, 0}$ into the expressions (2.22) for the resolvents

$$
\begin{aligned}
T(z) & =\frac{N+w_{\alpha}(z) w^{\alpha}(z) / m}{\sqrt{z^{2}-4 S / m}}=\frac{N}{\sqrt{z^{2}-4 S / m}}+\frac{w_{\alpha, 0} w_{0}^{\alpha}}{m\left(z^{2}-4 S / m\right)^{\frac{3}{2}}}, \\
w_{\alpha}(z) & =\frac{w_{\alpha, 0}}{\sqrt{z^{2}-4 S / m}}, \\
R(z) & =\frac{m}{2}\left(z-\sqrt{z^{2}-4 S / m}\right) .
\end{aligned}
$$

We can write $T(z)$ more compactly in terms of $\tilde{S}=S+\frac{1}{2 N} w_{\alpha, 0} w_{0}^{\alpha}$, the $S U(N)$ part of $S$

$$
T(z)=\frac{N}{\sqrt{z^{2}-4 \tilde{S} / m}} .
$$

Hence, $T(z)$ does not depend on the $U(1)$ photinos. It is easy to check (6.2) by expanding in $w_{\alpha, 0} w_{0}^{\alpha}$ and using the fact that higher order terms are zero by anticommutativity since $w_{\alpha}, 0$ is a two-component spinor. We substitute

$$
\int T(z)=N \ln \left(\frac{z+\sqrt{z^{2}-4 \tilde{S} / m}}{2}\right)
$$

into $(2.55)$ to find

$$
P(z)=\left(\frac{z+\sqrt{z^{2}-4 \tilde{S} / m}}{2}\right)^{N}+\frac{\Lambda^{2 N}}{\left(\frac{z+\sqrt{z^{2}-4 \tilde{S} / m}}{2}\right)^{N}}
$$


The chiral ring relations come from setting the negative powers of $z$ in the right hand side of (6.4) to zero. These relations are generated by

$$
\tilde{S}^{N}=m^{N} \Lambda^{2 N}
$$

or equivalently in terms of the $U(N)$ gaugino bilinear

$$
S^{N}+\frac{1}{2} S^{N-1} w_{\alpha, 0} w_{0}^{\alpha}=m^{N} \Lambda^{2 N}
$$

Hence we find that $P(z)$ is the Chebychev polynomial

$$
P(z)=\left(\frac{z+\sqrt{z^{2}-4 \tilde{S} / m}}{2}\right)^{N}+\left(\frac{z-\sqrt{z^{2}-4 \tilde{S} / m}}{2}\right)^{N}
$$

in agreement with [11].

The quantum relation (6.5) implies that in any vacuum $\left\langle\tilde{S}^{N}\right\rangle=m^{N} \Lambda^{2 N}$. Since the expectation values of products of chiral operators factorize

$$
\langle\tilde{S}\rangle^{N}=m^{N} \Lambda^{2 N}
$$

Solving for $\langle S\rangle$ we get $\langle S\rangle=\omega m \Lambda^{2}$ where $\omega$ is an $N^{\prime}$ th root of unity. We see that each of the $N$ solutions to the chiral ring relations corresponds to a supersymmetric vacuum with nonzero gaugino condensate, as claimed. The equations for photinos $w_{\alpha, i}$ depend on one independent fermion $w_{\alpha, 0}$, whence each of the massive vacua can have an arbitrary coherent state of the $U(1)$ photinos. The photon is massless by supersymmetry and the low energy gauge group is $U(1)$.

To find the expectation values of operators in each vacuum, we expand (6.1) in powers of $1 / z$. The odd moments vanish by the symmetry $\Phi \rightarrow-\Phi$ while the even moments are nonzero,

$$
\begin{aligned}
u_{2 k} & =N\left(\begin{array}{c}
2 k \\
k
\end{array}\right)(\tilde{S} / m)^{k}, \\
w_{\alpha, 2 k} & =w_{\alpha, 0}\left(\begin{array}{c}
2 k \\
k
\end{array}\right)(S / m)^{k}, \\
r_{2 k} & =\frac{S}{k+1}\left(\begin{array}{c}
2 k \\
k
\end{array}\right)(S / m)^{k} .
\end{aligned}
$$

The vacua are symmetrically distributed around zero in the complex $S$ plane. This pattern is reminiscent of the pure $\mathcal{N}=1$ supersymmetric $U(N)$ gauge theory. Indeed, 
we recover the chiral ring of the $\mathcal{N}=1 U(N)$ gauge theory by taking the mass $m$ of the adjoint field to infinity while holding the gaugino condensate $S$ and the strong coupling scale of the pure $U(N)$ gauge theory fixed

$$
\Lambda_{p}^{3 N}=m^{N} \Lambda^{2 N}
$$

The higher moments of $T(z), w_{\alpha}(z)$ and $R(z)$ vanish in the $m \rightarrow \infty$ limit $(6.9)$. The ring of the pure $U(N)$ gauge theory is generated by $S$ and $w_{\alpha, 0}$ which satisfy the relation

$$
S^{N}+\frac{1}{2} S^{N-1} w_{\alpha, 0} w_{0}^{\alpha}=\Lambda_{p}^{3 N}
$$

Let us now determine the classical ring. We see from the classical equations of motion

$$
W^{\prime}(\Phi)=m \Phi=0
$$

that $\Phi$ is a zero matrix. It follows that $u_{k}, w_{\alpha, k}$ and $r_{k}$ are zero in the ring for $k \geq 1$ because they contain $\Phi$. Hence $S$ and $w_{\alpha, 0}$ are the only nonzero operators. They are not constrained by the equations of motion. $S$ satisfies the relation

$$
S^{N}+\frac{1}{2} S^{N-1} w_{\alpha, 0} w^{\alpha, 0}=0
$$

which is the generalization of (5.3) when $\operatorname{Tr} W_{\alpha}$ is nonzero. It can be obtained by substituting $\tilde{S}$ for $S$ in (5.3). We notice the different origin of the formula for $S$ in the classical and the quantum chiral ring. Classically, (6.13) follows from the fermionic character of $W_{\alpha}$ while quantum mechanically (6.5) is a consequence of the anomaly equations together with the $\mathcal{N}=2$ relations.

\subsection{U(3) Gauge Theory with Cubic Superpotential}

We will now solve the chiral ring of the $U(3)$ gauge theory with cubic superpotential $W^{\prime}(z)=z^{2}-a z-b$ and show that it determines all the vacua of the theory. We will see that all vacua have nonzero gaugino condensation and that the chiral ring predicts the correct low energy gauge group. In this subsection, we will ignore the quadratic terms in the $U(1)$ photinos.

The polynomials $c(z), f(z)$ and $\rho_{\alpha}(z)$ are

$$
\begin{aligned}
c(z) & =2(z-a)+u_{1}, \\
f(z) & =-4\left((z-a) r_{0}+r_{1}\right), . \\
\rho_{\alpha}(z) & =(z-a) w_{0}+w_{1}
\end{aligned}
$$


For $U(3)$, the polynomials $P(z)$ and $P_{\alpha}(z)$ become

$$
\begin{gathered}
P(z)=z^{3}-z^{2} u_{1}+z\left(\frac{u_{1}^{2}-u_{2}}{2}\right)+\frac{3 u_{1} u_{2}-u_{1}^{3}-2 u_{3}}{6}, \\
P_{\alpha}(z)=\left(z^{2}-u_{1} z+\frac{u_{1}^{2}-u_{2}}{2}\right) w_{\alpha, 0}+\left(z-u_{1}\right) w_{\alpha, 1}+w_{\alpha, 2} .
\end{gathered}
$$

We get chiral ring relations by expanding (3.23) in $z$. Firstly, we express $u_{2}, r_{0}$ and $r_{1}$ in terms of $u_{1}$ and $u_{3}$

$$
\begin{aligned}
u_{2}= & 3 b+a u_{1}, \\
r_{0}= & -\frac{1}{6}\left(3 a b+\left(a^{2}+b\right) u_{1}-u_{3}\right), \\
r_{1}= & -\frac{1}{36}\left(-u_{1}^{4}+6 a u_{1}^{3}+u_{1}^{2}\left(-5 a^{2}+16 b\right)\right. \\
& \left.-6 u_{1}\left(3 a b+u_{3}\right)+6 a u_{3}-9 b^{2}\right) .
\end{aligned}
$$

Then $u_{3}$ can be found in terms of $u_{1}$ from the following equations

$$
\begin{array}{r}
\left(2 a^{2}-b-3 a u_{1}+u_{1}^{2}\right)\left(27 b u_{1}+9 a u_{1}^{2}-2 u_{1}^{3}-9 u_{3}\right)=0, \\
\left(\left(-91 a^{2}+77 b\right) u_{1}^{4}+39 a u_{1}^{5}-5 u_{1}^{6}+3 u_{1}^{3}\left(25 a^{3}-98 a b-6 u_{3}\right)\right. \\
+27 u_{1}^{2}\left(13 a^{2} b-5 b^{2}+2 a u_{3}\right)-9 u_{1}\left(-45 a b^{2}+8 a^{2} u_{3}+2 b u_{3}\right) \\
\left.+9\left(9 b^{3}-72 \Lambda^{6}-12 a b u_{3}+2 u_{3}^{2}\right)\right)=0 .
\end{array}
$$

$u_{1}$ is a solutions of the eight order polynomial equation

$$
\left(2 a^{2}-b-3 a u_{1}+u_{1}^{2}\right)\left(5832 \Lambda^{6}+\left(9 b+3 a u_{1}-u_{1}^{2}\right)^{3}\right)=0 .
$$

The relations for the gaugino operators $w_{\alpha, 0,1,2}$ are

$$
\begin{gathered}
\left(a u_{1}-u_{2}\right) w_{\alpha, 0}-3 a w_{\alpha, 1}+3 w_{\alpha, 2}=0 \\
\frac{1}{2}\left(u_{1}^{2}-u_{2}\right)\left(a w_{\alpha, 0}-w_{\alpha, 1}\right)+\left(u_{1}-3 a\right)\left(\frac{1}{2}\left(u_{1}^{2}-u_{2}\right) w_{\alpha, 0}-u_{1} w_{\alpha, 1}+w_{\alpha, 2}\right)=0 .
\end{gathered}
$$

After some algebraic manipulations using the equations (6.16) to (6.18), we find that product of any three gaugino bilinears can be written with the $\Lambda^{6}$ prefactor

$$
\begin{aligned}
r_{0}^{3}= & \frac{1}{6} \Lambda^{6}\left(6 a^{2}-6 a b-\left(10 a^{2}+b\right) u_{1}+3 a u_{1}^{2}+u_{3}\right), \\
r_{0}^{2} r_{1}= & -\frac{1}{18} \Lambda^{6}\left(a-u_{1}\right)\left(9 a^{2} u_{1}-16 a u_{1}^{2}+5 u_{1}^{3}+3 u_{3}\right), \\
r_{0} r_{1}^{2}= & \frac{1}{36} \Lambda^{6}\left(14 u_{1}^{5}-77 a u_{1}^{4}+2\left(74 a^{2}-25 b\right) u_{1}^{3}+\left(-121 a^{3}+146 a b+6 u_{3}\right) u_{1}^{2}\right. \\
& \left.+6\left(6 a^{4}-22 a^{2} b+5 b^{2}-2 a u_{3}\right) u_{1}+3 a\left(12 a^{2} b-9 b^{2}+2 a u_{3}\right)\right), \\
r_{1}^{3}= & \frac{1}{24} \Lambda^{6}\left(11 u_{1}^{6}-73 a u_{1}^{5}+5\left(37 a^{2}-9 b\right) u_{1}^{4}\right. \\
& +\left(-227 a^{3}+190 a b+4 u_{3}\right) u_{1}^{3}+\left(136 a^{4}-293 a^{2} b+33 b^{2}-12 a u_{3}\right) u_{1}^{2} \\
& \left.+a\left(-32 a^{4}+196 a^{2} b-69 b^{2}+12 a u_{3}\right) u_{1}-3 b\left(16 a^{4}-12 a^{2} b+b^{2}\right)\right) .
\end{aligned}
$$


Hence, by (5.24) and (6.20), the product of any three $r_{i}$ 's can be written as

$$
r_{i_{1}} r_{i_{2}} r_{i_{3}}=\Lambda^{6 l} Q_{i_{1}, i_{2}, i_{3}}^{l} .
$$

To keep the equations simple, we will continue the discussion for the superpotential $W^{\prime}(z)=z^{2}-z$. The equation for $u_{1}$ becomes

$$
\left(u_{1}-1\right)\left(u_{1}-2\right)\left(5832 \Lambda^{6}-u_{1}^{3}\left(u_{1}-3\right)^{3}\right)=0 .
$$

We will now discuss in detail all the roots to show each of them gives a supersymmetric vacuum of the gauge theory.

We see from (6.19) that the constrains for the expectation values of photinos are

$$
\begin{aligned}
w_{\alpha, 2}-w_{\alpha, 1} & =0 \\
u_{1}\left(u_{1}-1\right)\left(u_{1}-2\right) w_{\alpha, 0}-3\left(u_{1}-1\right)\left(u_{1}-2\right) w_{1} & =0 .
\end{aligned}
$$

For the vacua with $u_{1}=1,2 w_{\alpha, 1}, w_{\alpha, 2}$ can take arbitrary expectation values, they are massless. Hence, by supersymmetry the corresponding photons are massless as well and we have $U^{2}(1)$ low energy gauge group. The $w_{\alpha, 0}$ must have zero expectation value, it is massive. The remaining six vacua have only one massless direction for the photinos. Their low energy gauge group is $U(1)_{\text {free }}$.

The theory has four vacua coming from the confined $S U(2)$. Two of them are at $u_{1}=1$ and the other two at $u_{1}=2$. The two vacua with the same $u_{1}$ differ by the sign of gaugino condensate

$$
r_{0}=S= \pm \Lambda^{2} .
$$

Their positions in the $\mathcal{N}=2$ moduli space are distinguished by $u_{3}=u_{1}-6 r_{0}$. The two vacua at $u_{1}=1$ have $r_{1}=\operatorname{Tr} W^{2} \Phi=0$ because the gaugino condensation is in the $S U(2)$ part of the gauge group which is preserved $(0,0)$ block of $\Phi=\operatorname{diag}(0,0,1)$. The vacua $u_{1}=2$ have $r_{1}=r_{0}$ since the gauginos condense in the $(1,1)$ block of $\Phi=\operatorname{diag}(0,1,1)$. There are six vacua with confined $S U(3)$ that are symmetrically distributed in the $u_{1}$ plane around $u_{1}=0$ and around $u_{1}=3$,

$$
u_{1}=3 / 2 \pm \sqrt{3 / 2+18 \omega \Lambda^{2}}
$$

where $\omega$ is a third root of unity. As discussed in previous paragraph, these vacua have $U(1)_{\text {free }}$ gauge symmetry. All these vacua have nonzero gaugino condensation

$$
r_{0} \sim \Lambda^{2}
$$


with dominant one instanton contribution for small $\Lambda$. The vacua near $u_{1}=3$ with $\Phi=\operatorname{diag}(1,1,1)$ have the first moment of gaugino condensate of the same order $r_{1} \sim \Lambda^{2}$ as $r_{0}$. The vacua near $u_{1}=0$ with $\Phi_{\text {class }}=\operatorname{diag}(0,0,0)$ have vanishing one instanton contribution but nonzero second instanton contribution to $r_{1} \sim \Lambda^{4}$.

\section{Acknowledgements}

Firstly I would like to thank my advisor E. Witten for suggesting the problem and invaluable guidance and help throughout every stage of the project. I would also like to thank F. Cachazo, N. Seiberg for useful discussions. This research is supported in part by NSF grants PHY-9802484 and PHY-0243680. Any opinions, findings and conclusions or recommendations expressed in this material are those of the authors and do not necessarily reflect the views of the National Science Foundation.

\section{Appendix A.}

In this appendix, we will write for illustration first few of the $\mathcal{N}=2$ recursion formulas. These relations are expressed in terms of the characteristic polynomials $P(z)$ and $P_{m}(z)$. The coefficients of

$$
P(z)=\operatorname{det}(z-\Phi)=\prod_{i=1}^{N}\left(z-\lambda_{i}\right)=\sum_{i=0}^{N} p_{i} z^{N-i}
$$

are $p_{0}=1$ and $p_{k}=\sum_{i=1}^{N}(-1)^{k} \lambda_{i}^{k}$ for $k=1 \ldots N$, which can be expressed in terms of $u_{1}, \ldots, u_{N}$ from the recursion relations

$$
p_{k}=-\sum_{i=1}^{k} \frac{u_{i}}{k} p_{k-i}
$$

The first few $p_{i}$ 's are

$$
\begin{aligned}
& p_{0}=1, \\
& p_{1}=-u_{1}, \\
& p_{2}=-\frac{u_{2}}{2}+\frac{u_{1}^{2}}{2}, \\
& p_{3}=-\frac{u_{3}}{3}+\frac{u_{2} u_{1}}{3}-\frac{1}{6}\left(u_{1}^{2}-u_{2}\right) u_{1} .
\end{aligned}
$$

The characteristic polynomial $P_{m}(z)(2.28)$ comes from the first variation of $\delta \Phi=\epsilon M$ of $P(z)$

$$
P_{m}(z)=-\left.\partial_{\epsilon} \operatorname{det}(z-\Phi-\epsilon M)\right|_{\epsilon=0}=\sum_{i=1}^{N} M_{i i} \prod_{i \neq j}\left(z-\lambda_{i}\right)=\sum_{i=0}^{N-1} p_{m, i} z^{N-1-i} .
$$


We find the recursion relations for the coefficients $p_{m, k}=\sum_{i=1}^{N} M_{i i} \lambda_{i}^{k}$ by making the first order variation $\delta p_{k}=-p_{m, k-1}$ and $\delta u_{k}=k m_{k-1}$ of the recursion relation (A.2)

$$
p_{m, k}=\sum_{i=0}^{k} \frac{i}{k} m_{i} p_{k-i}-\sum_{i=1}^{k} \frac{1}{k} p_{m, k-i} u_{i} .
$$

The recursion relations together with first coefficient $p_{m, 0}=m_{0}$ determine $p_{m, k}$ in terms of $m_{1}, \ldots, m_{k}$ and $u_{1}, \ldots, u_{k}$. We write down the first few coefficients $p_{m, i}$ that are used in the examples

$$
\begin{aligned}
& p_{m, 0}=m_{0} \\
& p_{m, 1}=m_{1}-u_{1} m_{0} \\
& p_{m, 2}=m_{2}-u_{1} m_{1}+\frac{1}{2}\left(u_{1}^{2}-u_{2}\right) m_{0} .
\end{aligned}
$$

We are ready to show first few $\mathcal{N}=2$ relations obtained by expanding $(2.38)$ and (2.49) in powers of $1 / z$

$$
\begin{aligned}
T(z) & =\frac{P^{\prime}(z)}{\sqrt{P^{2}(z)-4 \Lambda^{2 N}}}, \\
w_{\alpha} & =\frac{P_{\alpha}(z)}{\sqrt{P^{2}(z)-4 \Lambda^{2 N}}}, \\
R(z) & =-\frac{P_{\alpha}(z) P^{\alpha}(z)}{2 P^{\prime}(z) \sqrt{P^{2}(z)-4 \Lambda^{2 N}}} .
\end{aligned}
$$

Let us note that in the last formula we are ignoring the part of the gaugino condensate that depends on the superpotential. The classical formulas are obtained by setting $\Lambda$ to zero in the quantum formulas.

For $U(2)$, all $u_{i}$ 's can be written as polynomials in $\Lambda^{4}$ and $u_{1}, u_{2}$ which are the two independent chiral operators that we can make from a $2 \times 2$ matrix $\Phi$. We have

$$
\begin{aligned}
& u_{3}=-\frac{1}{2}\left(u_{1}-3 u_{1} u_{2}\right) \\
& u_{4}=4 \Lambda^{4}-\frac{1}{2}\left(u_{1}^{4}-2 u_{1}^{2} u_{2}-u_{2}^{2}\right) \\
& u_{5}=10 u_{1} \Lambda^{4}-\frac{1}{4}\left(u_{1}^{5}-5 u_{1} u_{2}^{2}\right) .
\end{aligned}
$$

For $U(3)$, the first three $u_{1}, u_{2}$ and $u_{3}$ are independent. The higher moments are polynomials in these and in $\Lambda^{6}$

$$
\begin{aligned}
& u_{4}=\frac{1}{6}\left(u_{1}^{4}-6 u_{1}^{2} u_{2}+4 u_{2}^{2}+8 u_{1} u_{3}\right) \\
& u_{5}=\frac{1}{6}\left(u_{1}^{5}-5 u_{1}^{3} u_{2}+5 u_{1}^{2} u_{3}+5 u_{2} u_{3}\right) \\
& u_{6}=6 \Lambda^{6}+\frac{1}{12}\left(u_{1}^{6}-3 u_{1}^{4} u_{2}-9 u_{1}^{2} u_{2}^{2}+3 u_{2}^{3}+4 u_{1}^{3} u_{3}+12 u_{1} u_{2} u_{3}+4 u_{3}^{2}\right) .
\end{aligned}
$$


Taking $M=\frac{1}{4 \pi} W_{\alpha}$ in (A.4) to (A.6) we can read off the formulae for $w_{\alpha, i}$ from the $1 / z$ expansion of the generating relation (A.7). For $U(2)$, we find $w_{\alpha, i}$ 's as polynomials in $w_{\alpha, 0}, w_{\alpha, 1}$ and $u_{1}, u_{2}$

$$
\begin{aligned}
& w_{\alpha, 2}=-\frac{1}{2}\left(u_{1}^{2}-u_{2}\right) w_{\alpha, 0}+u_{1} w_{\alpha, 1} \\
& w_{\alpha, 3}=-\frac{1}{2}\left(u_{1}^{3}-u_{1} u_{2}\right) w_{\alpha, 0}+\frac{1}{2}\left(u_{1}^{2}+u_{2}\right) w_{\alpha, 1} \\
& w_{\alpha, 4}=2 \Lambda^{4} w_{\alpha, 0}-\frac{1}{4}\left(u_{1}^{4}-u_{2}^{2}\right) w_{\alpha, 0}+u_{1} u_{2} w_{\alpha, 1}
\end{aligned}
$$

The first few relations for $U(3)$ are

$$
\begin{aligned}
& w_{\alpha, 3}=\frac{1}{6}\left(u_{1}^{3}-3 u_{1} u_{2}+2 u_{3}\right) w_{\alpha, 0}-\frac{1}{2}\left(u_{1}^{2}-u_{2}\right) w_{\alpha, 1}+u_{1} w_{\alpha, 2} \\
& w_{\alpha, 4}=\frac{1}{6}\left(u_{1}^{4}-3 u_{1}^{2} u_{2}+2 u_{1} u_{3}\right) w_{\alpha, 0}-\frac{1}{3}\left(u_{1}^{2}-u_{3}\right) w_{\alpha, 1}+\frac{1}{2}\left(u_{1}^{2}+u_{2}\right) w_{\alpha, 2} .
\end{aligned}
$$

The relations for $R(z)$ give the infinitensimal gaugino condensate coming from the vacuum expectation value of photinos. Notice that are ignoring here the finite gaugino condensate that is induced by the superpotential (2.49). For $U(2)$, we have

$$
\begin{aligned}
& r_{0}=-\frac{1}{4} w_{\alpha, 0} w_{0}^{\alpha} \\
& r_{1}=\frac{1}{8} u_{1} w_{\alpha, 0} w_{0}^{\alpha}-\frac{1}{2} w_{\alpha, 0} w_{1}^{\alpha} \\
& r_{2}=\frac{1}{16}\left(u_{1}^{2}-3 u_{2}\right) w_{\alpha, 0} w_{0}^{\alpha}-\frac{1}{4} u_{1} w_{\alpha, 0} w_{1}^{\alpha}-\frac{1}{4} w_{\alpha, 1} w_{1}^{\alpha} .
\end{aligned}
$$

The first few cases for $U(3)$ are

$$
\begin{aligned}
& r_{0}=-\frac{1}{6} w_{\alpha, 0} w_{0}^{\alpha} \\
& r_{1}=\frac{1}{18} u_{1} w_{\alpha, 0} w_{0}^{\alpha}-\frac{1}{3} w_{\alpha, 0} w^{\alpha, 1} \\
& r_{2}=-\frac{1}{54}\left(u_{1}^{2}-3 u_{2}\right) w_{\alpha, 0} w_{0}^{\alpha}+\frac{1}{9} u_{1} w_{\alpha, 0} w_{1}^{\alpha}-\frac{1}{6} w_{\alpha, 1} w_{1}^{\alpha}-\frac{1}{3} w_{\alpha, 0} w_{2}^{\alpha} .
\end{aligned}
$$




\section{References}

[1] C. Vafa and N.P. Warner, "Catastrophes and the classification of conformal theories," Phys. Lett. B 218, 51 (1989) ; W. Lerche, C. Vafa and N. P. Warner, "Chiral Rings in N=2 Superconformal Theories," Nucl. Phys. B 324, 437 F(1989).

[2] E. Witten, "The Verlinde Algebra And The Cohomology Of The Grassmannian," arXiv:hep-th/9312104, and in Quantum Fields And Strings: A Course For Mathematicians, ed. P. Deligne et. al. (American Mathematical Society, 1999), vol. 2, pp. 1338-9.

[3] F. Cachazo, M. R. Douglas, N. Seiberg and E. Witten, "Chiral rings and anomalies in supersymmetric gauge theory," arXiv:hep-th/0211170.

[4] F. Cachazo and C. Vafa, "N = 1 and $\mathrm{N}=2$ geometry from fluxes," arXiv:hepth/0206017.

[5] F. Cachazo, N. Seiberg and E. Witten, "Phases of $N=1$ supersymmetric gauge theories and matrices," arXiv:hep-th/0301006.

[6] T. W. Hungerford, Algebra (Springer-Verlag, 1974).

[7] N. Seiberg and E. Witten, "Electric - magnetic duality, monopole condensation, and confinement in N=2 supersymmetric Yang-Mills theory," Nucl. Phys. B 426, 19 (1994) [Erratum-ibid. B 430, 485 (1994)] arXiv:hep-th/9407087.

[8] F. Cachazo, K. A. Intriligator and C. Vafa, "A large N duality via a geometric transition," Nucl. Phys. B 603, 3 (2001) arXiv:hep-th/0103067.

[9] J. de Boer and Y. Oz, "Monopole Condensation and Confining Phase of $\mathcal{N}=1$ Gauge Theories Via M Theory Fivebrane," arXiv:hep-th/9708044.

[10] E. Witten, "Chiral Ring Of $\mathrm{Sp}(\mathrm{N})$ and $\mathrm{SO}(\mathrm{N})$ Supersymmetric Gauge Theory In Four Dimensions." arXiv:hep-th/0302194

[11] M. R. Douglas and S. H. Shenker, "Dynamics of SU(N) supersymmetric gauge theory," Nucl. Phys. B 447, 271 (1995) arXiv:hep-th/9503163. 